\title{
Improved lower bounds on genuine-multipartite-entanglement concurrence
}

\author{
Zhi-Hua Chen, ${ }^{1}$ Zhi-Hao Ma,,${ }^{2}$ Jing-Ling Chen, ${ }^{3}$ and Simone Severini ${ }^{4}$ \\ ${ }^{1}$ Department of Science, Zhijiang college, Zhejiang University of technology, Hangzhou, 310024, P.R.China. \\ ${ }^{2}$ Department of Mathematics, Shanghai Jiao-Tong University, Shanghai, 200240, \\ P. R. China; currently visiting the Department of Physics 83 Astronomy, \\ University College London, WC1E 6BT London, United Kingdom; \\ ${ }^{3}$ Theoretical Physics Division, Chern Institute of Mathematics, Nankai University, \\ Tianjin, 300071, P. R.China; Centre for Quantum Technologies, \\ National University of Singapore, 3 Science Drive 2, 117543 \\ ${ }^{4}$ Department of Computer Science, and Department of Physics \& Astronomy, \\ University College London, WC1E 6BT London, United Kingdom.
}

\begin{abstract}
Genuine-multipartite-entanglement (GME) concurrence is a measure of genuine multipartite entanglement that generalizes the well-known notion of concurrence. We define an observable for GME concurrence. The observable permits us to avoid full state tomography and leads to different analytic lower bounds. By means of explicit examples we show that entanglement criteria based on the bounds have a better performance with respect to the known methods.
\end{abstract}

PACS numbers:

\section{INTRODUCTION}

Entanglement plays a fundamental role in the study of many-body quantum mechanics. Complex systems with multipartite quantum correlations have been shown to be useful in numerous tasks, ranging from measurement based quantum computing [1], quantum secret sharing [2], quantum communication [3], etc. Compared with the bipartite case, multipartite entanglement is well-known to exhibit richer structures and a variety of classes (see, e.g., [4, 5] ). Being substantially different from partial entanglement (i.e., entanglement specified by correlations between any two subsystems), the so-called genuine multipartite entanglement (for short, GME) is of special interest. Although many efforts have been devoted towards the detection of GME (e.g., entanglement witnesses [6], Bell-like inequalities [7], etc.), its characterization still remains a difficult problem [8]. On the other hand, the quantitative aspects are important because these are justified by the experimental perspective [9].

While the three-tangle is a famous measure of GME for three qubits [10], no such a concept is currently available for systems of higher dimension. Recently, a notion of generalized concurrence, called GME-concurrence [11], was introduced in the attempt of distinguishing between GME and partial entanglement. In the present paper, we first point out that GME concurrence of pure states may be directly accessible in laboratory experiments (i.e., no full state tomography is needed) provided that a two-fold copy of the state is available. Then, we present some explicit lower bounds. We illustrate detailed examples in which the given bounds perform better when compared with other known detection criteria. The evaluation of the bounds permit to bypass full quantum tomography, since we only need a polynomial number (on the system's dimension) of expectation values. The results appear to be an improvement over [11].

The remainder of the paper is organized as follows. In Section 2, we recall the definition and the basic properties of GME-concurrence. In Section 3, we show that GME-concurrence is an observable measure. In Section 4, we state and prove the bounds. Section 5 is devoted to examples. Section 6 contains some brief conclusions.

\section{GME-CONCURRENCE}

An $N$-partite pure state $|\phi\rangle$ with Hilbert space $\mathcal{H}_{1} \otimes \mathcal{H}_{2} \otimes \cdots \otimes \mathcal{H}_{N}$ is said to be biseparable if there is a bipartition $\gamma \mid \gamma^{\prime}$ such that $|\phi\rangle$ can be decomposed as a tensor product $\left|\phi_{\gamma \mid \gamma^{\prime}}\right\rangle=\left|\phi_{\gamma}\right\rangle \otimes\left|\phi_{\gamma^{\prime}}\right\rangle$. If an $N$-partite pure state is not biseparable then it is said to be genuinely $N$-partite entangled. The same terms apply to an $N$-partite mixed state $\rho$, if it can (resp. it can not) be written as a convex combination of biseparable pure states $\rho=\sum_{i} p_{i}\left|\phi_{\gamma_{i} \mid \gamma_{i}^{\prime}}\right\rangle\left\langle\phi_{\gamma_{i} \mid \gamma_{i}^{\prime}}\right.$, where

*Electronic address: ma9452316@gmail.com 
each component $\left|\phi_{\gamma_{i} \mid \gamma_{i}^{\prime}}\right\rangle$ is biseparable (possibly under different partitions). Given an $N$-partite pure state $|\phi\rangle$, let $\gamma=\left\{j_{1}, j_{2}, \ldots, j_{k}\right\} \subseteq\{1,2, \ldots, N\}$ be a subset inducing a bipartition $j_{1}, j_{2}, \ldots, j_{k} \mid j_{k+1}, \ldots, j_{N}$. If $C_{\gamma}^{2}(\phi):=1-\operatorname{Tr}\left(\rho_{\gamma}^{2}\right)$, where $\rho_{\gamma}$ is the reduced density matrix of the subsystem indexed by $\gamma$, the GME-concurrence (of a pure state) is

$$
C_{G M E}(\phi):=\sqrt{\min _{\gamma} C_{\gamma}^{2}(\phi)}
$$

For example, let us consider a three-qubit state $|\phi\rangle$. In this case, we have $\gamma=\{1\}, \gamma=\{2\}$ or $\gamma=\{3\}$, corresponding to the partitions $1|2,3,2| 1,3$, and $3 \mid 1,2$, respectively. Its GME-concurrence is then

$$
C_{G M E}^{2}(\phi)=\min _{\gamma=\{1\},\{2\},\{3\}}\left\{1-\operatorname{Tr}\left(\rho_{1}^{2}\right), 1-\operatorname{Tr}\left(\rho_{2}^{2}\right), 1-\operatorname{Tr}\left(\rho_{3}^{2}\right)\right\}
$$

More generally, the GME-concurrence of an $N$-partite mixed state $\rho$ is

$$
C_{G M E}(\rho):=\min \sum_{i} p_{i} C_{G M E}\left(\phi_{i}\right)
$$

where the minimum is taken over all pure states decompositions $\rho=\sum_{i} p_{i}\left|\phi_{i}\right\rangle\left\langle\phi_{i}\right|$. It is worth recalling that GMEconcurrence satisfies the following useful properties [11]:

M1. The GME-concurrence is zero for all biseparable states;

M2. The GME-concurrence is strictly greater than zero for all GME states;

M3. (Convexity) $C_{G M E}\left(\sum_{i} p_{i} \rho_{i}\right) \leq \sum_{i} p_{i} C_{G M E}\left(\rho_{i}\right)$;

M4. (Non-increasing under LOCC) $C_{G M E}\left(\Lambda_{L O C C}(\rho)\right) \leq C_{G M E}(\rho)$;

M5. (Invariance under local unitary transformations) $C_{G M E}\left(U_{\text {local }} \rho U_{\text {local }}^{+}\right)=C_{G M E}(\rho)$;

M6. (Subadditivity) $C_{G M E}(\rho \otimes \sigma) \leq C_{G M E}(\rho)+C_{G M E}(\sigma)$.

\section{GME-CONCURRENCE OF PURE STATES IS OBSERVABLE}

In this section, we describe an observable for GME-concurrence of pure states. More specifically, GME-concurrence for pure state can be measured directly, provided that two copies of the state are available. Notice that our approach is quite different from that of [12], in particular, we do not use symmetric and antisymmetric projections subspace. As above, let $|\phi\rangle$ be an $N$-partite pure state with Hilbert space $\mathcal{H}_{1} \otimes \mathcal{H}_{2} \otimes \cdots \otimes \mathcal{H}_{N}$, of respective dimensions $d_{1}, d_{2}, \ldots, d_{N}$. We can write

$$
|\phi\rangle:=\sum_{i_{1}, i_{2}, \ldots, i_{N}} \phi_{i_{1} i_{2} \cdots i_{N}}\left|i_{1} i_{2} \cdots i_{N}\right\rangle,
$$

where $i_{j}$ is the $i$-th element of an orthonormal basis of $\mathcal{H}_{j}$, with $j=1, \ldots, N$. Given a subset $\gamma=\left\{t_{1}, t_{2}, \ldots, t_{k}\right\} \subseteq$ $\{1,2, \ldots, N\}$, the $\gamma$-concurrence of $\rho$ can be written as $C_{\gamma}^{2}(\phi)=\left\langle\phi\left|\otimes\left\langle\phi\left|B_{\gamma}\right| \phi\right\rangle \otimes\right| \phi\right\rangle$. The observable $B_{\gamma}$ is independent of $|\phi\rangle$ and it is uniquely determined by the partition induced by $\gamma$. The definition of $B_{\gamma}$ requires some preparation. Let $\gamma=\left\{t_{1}, t_{2}, \ldots, t_{k}\right\}$. Let

$$
I:=\left\{j_{1}, \ldots, j_{k}\right\} \cup\left\{j_{k+1}, j_{k+2}, \ldots, j_{N}\right\} \text { and } J^{\prime}:=\left\{j_{1}^{\prime}, \ldots, j_{k}^{\prime}\right\} \cup\left\{j_{k+1}^{\prime}, j_{k+2}^{\prime}, \ldots, j_{N}^{\prime}\right\}
$$

be two arbitrary index sets such that $j_{i}, j_{i}^{\prime}=0, \ldots, d_{t_{i}}-1$, with $t_{i}=1, \ldots, N$. Here, $\left\{j_{1}, \ldots, j_{k}\right\}$ and $\left\{j_{1}^{\prime}, \ldots, j_{k}^{\prime}\right\}$ indicate the same positions as the ones indexed by $\gamma$. For instance, for a three-qubit state, if $\gamma=\{2\}$ then $\left\{j_{1}, \ldots, j_{k}\right\}$ and $\left\{j_{1}^{\prime}, \ldots, j_{k}^{\prime}\right\}$ indicate elements in the second subsystem $\mathcal{H}_{2}$. We then define two further index subsets,

$$
I_{\gamma}:=\left\{j_{1}, \ldots, j_{k}\right\} \text { and } J_{\gamma}^{\prime}:=\left\{j_{1}^{\prime}, \ldots, j_{k}^{\prime}\right\}
$$

These subsets are obtained from $I, J^{\prime}$ and the partition $\gamma$. We also define the complements

$$
I \backslash I_{\gamma}:=\left\{j_{k+1}, j_{k+2}, \ldots, j_{N}\right\} \text { and } J^{\prime} \backslash J_{\gamma}^{\prime}:=\left\{j_{k+1}^{\prime}, j_{k+2}^{\prime}, \ldots, j_{N}^{\prime}\right\}
$$

Finally, we have the following two index sets obtained by swapping the elements in the positions corresponding to the ones indexed by $\gamma: I^{\prime}:=J_{\gamma}^{\prime} \cup I \backslash I_{\gamma}$ and 
$J:=I_{\gamma} \cup J^{\prime} \backslash J_{\gamma}^{\prime}$.

Once $I$ and $J^{\prime}$ are arbitrarily fixed, then $I^{\prime}$ and $J$ are uniquely determined by $\gamma$. With the use of this notation, we can finally write

$$
\begin{aligned}
C_{\gamma}^{2}(\phi) & =1-\operatorname{Tr}\left(\rho_{\gamma}^{2}\right)=\left(\sum_{i_{1}, i_{2}, \ldots, i_{N}} \phi_{i_{1} i_{2} \cdots i_{N}} \bar{\phi}_{i_{1} i_{2} \cdots i_{N}}\right)^{2} \\
& -\sum_{I_{\gamma}} \sum_{J_{\gamma}^{\prime}} \sum_{I \backslash I_{\gamma}} \phi_{I} \bar{\phi}_{I^{\prime}} \sum_{J^{\prime} \backslash J_{\gamma}^{\prime}} \phi_{J^{\prime}} \bar{\phi}_{J} \\
& =\sum_{I, J^{\prime}} \phi_{I} \bar{\phi}_{I} \phi_{J^{\prime}} \bar{\phi}_{J^{\prime}}-\sum_{I, J^{\prime}} \phi_{I} \bar{\phi}_{I^{\prime}} \phi_{J^{\prime}} \bar{\phi}_{J} \\
& =\sum_{I, J^{\prime}}\left(\phi_{I} \phi_{J^{\prime}}-\phi_{I^{\prime}} \phi_{J}\right)\left(\bar{\phi}_{I} \bar{\phi}_{J^{\prime}}-\bar{\phi}_{I^{\prime}} \bar{\phi}_{J}\right) \\
& =\sum_{I, J^{\prime}}\left|\left(\phi_{I} \phi_{J^{\prime}}-\phi_{I^{\prime}} \phi_{J}\right)\right|^{2}
\end{aligned}
$$

where the sum is taken over all possible index sets $I$ and $J^{\prime}$. The observable is

$$
\begin{aligned}
B_{\gamma} & =\sum_{i_{1}, i_{2}, \ldots, i_{N}}\left|i_{1} i_{2} \cdots i_{N}\right\rangle \otimes\left|i_{1} i_{2} \cdots i_{N}\right\rangle \\
& \times \sum_{i_{1}, i_{2}, \ldots, i_{N}}\left\langle i_{1} i_{2} \cdots i_{N}\right| \otimes\left\langle i_{1} i_{2} \cdots i_{N}\right| \\
& -\sum_{I_{\gamma}} \sum_{J_{\gamma}^{\prime}} \sum_{I \backslash I_{\gamma}}|I\rangle \otimes\left|I^{\prime}\right\rangle \sum_{I \backslash I_{\gamma}}\langle I| \otimes\left\langle I^{\prime}\right| .
\end{aligned}
$$

It follows that

$$
C_{\gamma}^{2}(\phi)=\sum_{I, J^{\prime}}\left|\left(\phi_{I} \phi_{J^{\prime}}-\phi_{I^{\prime}} \phi_{J}\right)\right|^{2}=\left\langle\phi\left|\otimes\left\langle\phi\left|B_{\gamma}\right| \phi\right\rangle \otimes\right| \phi\right\rangle,
$$

which gives a general expression for the GME-concurrence of a pure state:

$$
\begin{aligned}
C_{G M E}^{2}(\phi) & =\min _{\gamma}\left\langle\phi\left|\otimes\left\langle\phi\left|B_{\gamma}\right| \phi\right\rangle \otimes\right| \phi\right\rangle \\
& =\min _{\gamma}\left(\sum_{I, J, I^{\prime}, J^{\prime}}\left|\left(\phi_{I} \phi_{J^{\prime}}-\phi_{I^{\prime}} \phi_{J}\right)\right|^{2}\right) .
\end{aligned}
$$

For example, let

$$
|\phi\rangle=\sum_{i, j, k \in\{0,1\}} \phi_{i j k}|i j k\rangle
$$

be a generic three-qubit pure state. If $\gamma=\{1\}$ then

$$
\begin{aligned}
C_{1}^{2}(\phi) & =\left\langle\phi\left|\otimes\left\langle\phi\left|B_{1}\right| \phi\right\rangle \otimes\right| \phi\right\rangle \\
& =2\left(\left|\phi_{000} \phi_{101}-\phi_{100} \phi_{001}\right|^{2}+\left|\phi_{000} \phi_{110}-\phi_{100} \phi_{010}\right|^{2}\right. \\
& +\left|\phi_{000} \phi_{111}-\phi_{100} \phi_{011}\right|^{2} \\
& +\left|\phi_{001} \phi_{110}-\phi_{101} \phi_{010}\right|^{2}+\left|\phi_{001} \phi_{111}-\phi_{101} \phi_{011}\right|^{2} \\
& \left.+\left|\phi_{010} \phi_{111}-\phi_{110} \phi_{011}\right|^{2}\right)
\end{aligned}
$$

and the observable is

$$
\begin{aligned}
B_{1} & \left.=\sum_{i, j, k \in\{0,1\}}|i j k| i j k\right\rangle \sum_{i, j, k \in\{0,1\}}\langle i j k|i j k| \\
& \left.-\sum_{i \in\{0,1\}} \sum_{i^{\prime} \in\{0,1\}} \sum_{j, k}|i j k| i^{\prime} j k\right\rangle \sum_{j, k}\left\langle i j k\left|i^{\prime} j k\right| .\right.
\end{aligned}
$$

The observables $B_{2}$ and $B_{3}$ are obtained analogously. 


\section{LOWER BOUNDS}

\section{A. Statement of the results}

Let $|\psi\rangle=\bigotimes_{i=1}^{N}\left|x_{i}\right\rangle=\left|x_{1} x_{2} \cdots x_{N}\right\rangle$ be a product state with Hilbert space $\mathcal{H}^{\prime} \mathcal{H}_{1} \otimes \mathcal{H}_{2} \otimes \cdots \otimes \mathcal{H}_{N} \cdot$ Let $\left|\psi_{i}\right\rangle=\left|x_{1} x_{2} \cdots x_{i-1} x_{i}^{\prime} x_{i+1} \cdots x_{N}\right\rangle$ and $\left|\psi_{j}\right\rangle=\left|x_{1} x_{2} \cdots x_{j-1} x_{j}^{\prime} x_{j+1} \cdots x_{N}\right\rangle$ be the product states obtained from $|\psi\rangle$ by applying (independently) local unitaries to $\left|x_{i}\right\rangle \in \mathcal{H}_{i}$ and $\left|x_{j}\right\rangle \in \mathcal{H}_{j}$, respectively. Let $\left|\Psi_{i j}\right\rangle:=\left|\psi_{i}\right\rangle\left|\psi_{j}\right\rangle$ be a product state on $\mathcal{H}^{\otimes 2}=\left(\mathcal{H}_{1} \otimes \mathcal{H}_{2} \otimes \cdots \otimes \mathcal{H}_{N}\right)^{\otimes 2}$. Let us define $\Pi=\mathcal{P}_{1} \circ \mathcal{P}_{2} \circ \cdots \circ \mathcal{P}_{N}$, where $\mathcal{P}_{i}$ is the operator swapping the two copies of $\mathcal{H}_{i}$ in $\mathcal{H}^{\otimes 2}$, for each $i=1, \ldots, N$. Finally, let $\rho$ be an arbitrary state in the total Hilbert space $\mathcal{H}$. We will prove the following statements:

Bound1. By writing

$$
\begin{aligned}
\mathcal{F}(\rho, \psi) & =\sum_{1 \leq i \neq j \leq N} \sqrt{\left\langle\Psi_{i j}\left|\rho^{\otimes 2} \Pi\right| \Psi_{i j}\right\rangle} \\
& -\sum_{1 \leq i \neq j \leq N} \sqrt{\left\langle\Psi_{i j}\left|\mathcal{P}_{i}^{\dagger} \rho^{\otimes 2} \mathcal{P}_{i}\right| \Psi_{i j}\right\rangle} \\
& -(N-2) \sum_{1 \leq i \leq N} \sqrt{\left\langle\Psi_{i i}\left|\mathcal{P}_{i}^{\dagger} \rho^{\otimes 2} \mathcal{P}_{i}\right| \Psi_{i i}\right\rangle}
\end{aligned}
$$

we have

$$
\mathcal{F}(\rho, \psi) \leq \sqrt{2}(N-1) \cdot C_{G M E}(\rho) .
$$

Bound2. For any given $|\psi\rangle$, we can get $\psi_{i}=\left|x_{1} \cdots x_{i-1} x_{i}^{\prime} x_{i+1} \cdots x_{j-1} x_{j} x_{j+1} \cdots x_{N}\right\rangle$ by changing the $i$ th bit of $|\psi\rangle$,Let $\left|\psi_{i j}\right\rangle=\left|x_{1} \cdots x_{i-1} x_{i}^{\prime} x_{i+1} \cdots x_{j-1} x_{j}^{\prime} x_{j+1} \cdots x_{N}\right\rangle$ by changing the $j$ th bit of $\left.\psi_{i}\right\rangle$, and $\Psi_{i_{l} i_{m}}:=\left|\psi_{i_{l}}\right\rangle\left|\psi_{i_{m}}\right\rangle$ be defined as above, but obtained by the application of two local unitaries. By writing

$$
\begin{aligned}
\mathcal{L}\left(\rho, \psi_{i}\right) & =\sum_{l \neq m, l \neq i, m \neq i} \sqrt{\left\langle\Psi_{i_{l} i_{m}}\left|\rho^{\otimes 2} \Pi\right| \Psi_{i_{l} i_{m}}\right\rangle} \\
& -\sum_{l \neq m, l \neq i, m \neq i} \sqrt{\left\langle\Psi_{i_{l} i_{m}}\left|\mathcal{P}_{l}^{\dagger} \rho^{\otimes 2} \mathcal{P}_{l}\right| \Psi_{i_{l} i_{m}}\right\rangle} \\
& -(N-3) \sum_{l \neq i} \sqrt{\left\langle\Psi_{i_{l} i_{l}}\left|\mathcal{P}_{l}^{\dagger} \rho^{\otimes 2} \mathcal{P}_{l}\right| \Psi_{i_{l} i_{l}}\right\rangle}
\end{aligned}
$$

we have

$$
\sum_{1 \leq i \leq N} \mathcal{L}\left(\rho, \psi_{i}\right) \leq 2 \sqrt{N-2} \cdot C_{G M E}(\rho) .
$$

Bound3. Let $V=\left\{\left|\chi_{1}\right\rangle, \ldots,\left|\chi_{m}\right\rangle\right\}$ be a set of product states in $\mathcal{H}$. Then

$$
\begin{aligned}
\mathcal{T}(\rho, \chi) & =\sum_{\mid \chi_{\alpha\rangle \in V}} \sum_{\left|\chi_{\beta}\right\rangle \in K_{\alpha}}\left(\left|\left\langle\chi_{\alpha}|\rho| \chi_{\beta}\right\rangle\right|\right. \\
& \left.-\sqrt{\left\langle\chi_{\alpha}\left|\otimes\left\langle\chi_{\beta}\left|\Pi_{\alpha \beta} \rho^{\otimes 2} \Pi_{\alpha \beta}\right| \chi_{\alpha}\right\rangle \otimes\right| \chi_{\beta}\right\rangle}\right) \\
& -\left(s-s_{0}\right) \sum_{\alpha}\left\langle\chi_{\alpha}|\rho| \chi_{\alpha}\right\rangle \\
& \leq \sqrt{2} s \cdot C_{G M E}(\rho)
\end{aligned}
$$

where

$$
\left.K_{\alpha}=\left\{\left|\chi_{\beta}\right\rangle:|| \chi_{\alpha}\right\rangle \cap\left|\chi_{\beta}\right\rangle \mid=N-2 \text { with }\left|\chi_{\alpha}\right\rangle,\left|\chi_{\beta}\right\rangle \in V\right\},
$$

and $s=\max \left|K_{\alpha}\right|$. Additionally,

$$
s_{0}=\min _{1 \leq i \leq N} s_{\alpha, i}
$$


where $s_{\alpha, i}$ is the number of the vectors in $K_{\alpha}$ such that the $i$-th bits of $v_{\alpha}$ are different when $K_{\alpha} \neq \emptyset$. We denote by $\left.\| v_{\alpha}\right\rangle \cap\left|v_{\beta}\right\rangle \mid$ the number of coordinates that are equal in both vectors. $\Pi_{\alpha \beta}$ swaps one different bit of $\left|\chi_{\alpha}\right\rangle$ and $\left|\chi_{\beta}\right\rangle$.

The definition of the witness in Bound 1 appeared in [13, 14]. When $m=2$ and $N=4$ the bound in Eq. (6) is the same as the criterion given in [15]. However, this is not always the case, as we shall verify below.

\section{B. Proof}

\section{Bound 1}

We start with a three-qubit state to get an intuition for the general case that we shall discuss later. We are interested in bounding the GME concurrence of an arbitrary three-qubit state $\rho=\sum_{i} p_{i} \phi^{(i)}$, with pure state decomposition $\left\{p_{i}, \phi^{(i)}\right\}$. We select a product state $|\psi\rangle=|001\rangle$. If we apply the bit flip operation to the $i$-th qubit, we have $\left|\psi_{1}\right\rangle=|101\rangle,\left|\psi_{2}\right\rangle=|011\rangle$, and $\left|\psi_{3}\right\rangle=|000\rangle$. The bound given in Eq. (5) is

$$
\begin{aligned}
\mathcal{F}(\rho, \psi) & =2\left(\left|\rho_{4,6}\right|+\left|\rho_{1,4}\right|+\left|\rho_{1,6}\right|-\sqrt{\rho_{2,2} \rho_{8,8}}\right. \\
& \left.-\sqrt{\rho_{2,2} \rho_{3,3}} \sqrt{\rho_{2,2} \rho_{5,5}}\right) \\
& -\rho_{1,1}-\rho_{4,4}-\rho_{6,6} \leq 2 \sqrt{2} \cdot C_{G M E}(\rho) .
\end{aligned}
$$

For proving this, let us consider the pure state $|\phi\rangle$ as in Eq. (3). With the use of the Cauchy-Schwarz and the triangle inequality, we obtain $C_{i}(\phi)$, for $i=1,2,3$ :

$$
\begin{aligned}
& \sqrt{2} C_{1}(\phi) \geq\left(\left|\phi_{011} \phi_{101}\right|-\left|\phi_{001} \phi_{111}\right|+\left|\phi_{000} \phi_{101}\right|-\left|\phi_{001} \phi_{100}\right|\right) \\
& \sqrt{2} C_{2}(\phi) \geq\left(\left|\phi_{000} \phi_{011}\right|-\left|\phi_{001} \phi_{010}\right|+\left|\phi_{011} \phi_{101}\right|-\left|\phi_{001} \phi_{111}\right|\right) \\
& \sqrt{2} C_{3}(\phi) \geq\left(\left|\phi_{000} \phi_{011}\right|-\left|\phi_{001} \phi_{010}\right|+\left|\phi_{000} \phi_{101}\right|-\left|\phi_{001} \phi_{100}\right|\right)
\end{aligned}
$$

By the same step,

$$
\begin{aligned}
\mathcal{F}(\phi, \psi) & =2\left(\left|\phi_{011} \phi_{101}\right|-\left|\phi_{001} \phi_{111}\right|+\left|\phi_{000} \phi_{011}\right|\right. \\
& \left.-\left|\phi_{001} \phi_{010}\right|+\left|\phi_{000} \phi_{101}\right|-\left|\phi_{001} \phi_{100}\right|\right) \\
& -\left(\left|\phi_{000}\right|^{2}+\left|\phi_{011}\right|^{2}+\left|\phi_{101}\right|^{2}\right) \\
& \leq 2 \sqrt{2} \min \left\{C_{1}(\phi), C_{2}(\phi), C_{3}(\phi)\right\} .
\end{aligned}
$$

This confirms the statement in Eq. (8), when restricted to pure states. If $\rho$ is a mixed state, the convex roof construction is bounded as

$$
2 \sqrt{2} C_{G M E}(\rho) \geq \inf _{\left\{p_{i},\left|\phi_{i}\right\rangle\right\}} \sum_{i} p_{i} \mathcal{F}\left(\phi^{(i)}, \psi\right)
$$

where $\left\{p_{i}, \phi^{(i)}\right\}$ is any pure state decomposition of $\rho$. Having chosen $|\psi\rangle=|001\rangle$, we obtain Eq. (8). Since $C_{G M E}(\rho)$ is invariant under local unitaries, for any choice of a product state $|\psi\rangle, \mathcal{F}(\rho, \psi)$ is a lower bound to $C_{G M E}(\rho)$ leading to $C_{G M E}(\rho) \geq \frac{1}{2} \mathcal{F}(\rho, \psi)$. This concludes the proof for the three-qubit case.

We are now ready to prove the inequality for a general $N$-qudit state. Some notation is needed:

$$
\begin{aligned}
c_{0} & :=x_{1} \cdots x_{i-1} x_{i} x_{i+1} \cdots x_{N} \\
c_{i} & :=x_{1} \cdots x_{i-1} x_{i}^{\prime} x_{i+1} \cdots x_{N} ; \\
c_{j} & :=x_{1} \cdots x_{j-1} x_{j}^{\prime} x_{j+1} \cdots x_{N} ; \\
c_{i j} & :=x_{1} \cdots x_{i-1} x_{i}^{\prime} x_{i+1} \cdots x_{j-1} x_{j}^{\prime} x_{j+1} \cdots x_{N} ;
\end{aligned}
$$

when $i<j$, we use $c_{i j}$; otherwise, we use $c_{j i}$.

Again, since $C_{G M E}(\rho)$ is invariant under local unitaries, we only need to consider the integers $0 \leq x_{i} \leq d_{i}-1$, for $i=1,2, \ldots, N$. For the generic $N$-qudit pure state $|\phi\rangle$ in Eq. (2), the bound in Eq. (4) reads as

$$
\mathcal{F}(\phi, \psi)=\sum_{1 \leq i \neq j \leq N}\left(\left|\phi_{c_{i}} \phi_{c_{j}}\right|-\left|\phi_{c_{0}} \phi_{c_{i j}}\right|\right)-(N-2) \sum_{1 \leq i \leq N}\left|\phi_{c_{i}}\right|^{2} .
$$


There are two cases depending on the biseparable partition $\gamma$ :

Case 1. For any given $\gamma \subset\{1,2, \ldots N\}$ with $|\{\gamma\}|=1$,

$$
\begin{aligned}
2 \sqrt{(N-1)} C_{\gamma}(\phi) & =2 \sqrt{(N-1)} \sqrt{\sum_{j \neq \gamma}\left|\phi_{c_{\gamma}} \phi_{c_{j}}-\phi_{c_{0}} \phi_{c_{\gamma j}}\right|^{2}} \\
& \geq \sum_{j \neq \gamma}\left|\phi_{c_{\gamma}} \phi_{c_{j}}-\phi_{c_{0}} \phi_{c_{\gamma j}}\right| \\
& \geq \sum_{j \neq \gamma}\left|\phi_{c_{\gamma}} \phi_{c_{j}}\right|-\left|\phi_{c_{0}} \phi_{c_{\gamma j}}\right|
\end{aligned}
$$

It is convenient to interpret $\mathcal{F}(\phi, \psi)$ as a sum of two terms:

$$
\begin{aligned}
\mathcal{F}(\phi, \psi) & =\sum_{j \neq \gamma}\left(\left|\phi_{c_{j}} \phi_{c_{\gamma}}\right|-\left|\phi_{c_{0}} \phi_{c_{j \gamma}}\right|\right) \\
& +\sum_{i \neq \gamma, j \neq \gamma}\left(\left|\phi_{c_{i}} \phi_{c_{j}}\right|-\left|\phi_{c_{0}} \phi_{c_{i j}}\right|\right)-(N-2) \sum_{1 \leq i \leq N}\left|\phi_{c_{i}}\right|^{2} \\
& =X+Y
\end{aligned}
$$

where

$$
\begin{aligned}
X & =\sum_{j \neq \gamma}\left(\left|\phi_{c_{j}} \phi_{c_{\gamma}}\right|-\left|\phi_{c_{0}} \phi_{c_{j \gamma}}\right|\right) \leq \sum_{j \neq \gamma}\left|\phi_{c_{\gamma}} \phi_{c_{j}}-\phi_{c_{0}} \phi_{c_{j \gamma}}\right| \\
& \leq 2 \sqrt{(N-1)} C_{\gamma}(\phi)
\end{aligned}
$$

and

$$
\begin{aligned}
Y & =\sum_{i \neq \gamma, j \neq \gamma}\left(\left|\phi_{c_{i}} \phi_{c_{j}}\right|-\left|\phi_{c_{0}} \phi_{c_{i j}}\right|\right)-(N-2) \sum_{1 \leq i \leq N}\left|\phi_{c_{i}}\right|^{2} \\
& \leq \sum_{i \neq \gamma, j \neq \gamma} \frac{\left|\phi_{c_{i}}\right|^{2}+\left|\phi_{c_{j}}\right|^{2}}{2}-(N-2) \sum_{1 \leq i \leq N}\left|\phi_{c_{i}}\right|^{2} \\
& =(N-2) \sum_{1 \leq i \leq N}\left|\phi_{c_{i}}\right|^{2}-(N-2) \sum_{1 \leq i \leq N}\left|\phi_{c_{i}}\right|^{2} \\
& =0 .
\end{aligned}
$$

Hence,

$$
\mathcal{F}(\phi, \psi) \leq \min _{\gamma=1,2, \cdots, N} 2 \sqrt{(N-1)} \cdot C_{\gamma}(\phi) .
$$

Case 2. For any given $\gamma=\left\{j_{1}, j_{2}, \ldots, j_{k}\right\} \subset\{1,2, \ldots N\}$, with $k \geq 2$,

$$
\begin{aligned}
& 2 \sqrt{(N-1)} \cdot C_{\gamma}(\phi) \\
& \geq 2 \sqrt{(N-k) k} \cdot C_{\gamma}(\phi) \\
& =2 \sqrt{(N-k) k} \sqrt{\sum_{l=1}^{k} \sum_{j \neq j_{t}: 1 \leq t \leq k}\left|\phi_{c_{j_{l}}} \phi_{c_{j}}-\phi_{c_{0}} \phi_{c_{j_{l} j}}\right|^{2}} \\
& \geq \sum_{l=1}^{k} \sum_{j \neq j_{t}: 1 \leq t \leq k}\left|\phi_{c_{j_{l}}} \phi_{c_{j}}\right|-\left|\phi_{c_{0}} \phi_{c_{j_{l} j}}\right| .
\end{aligned}
$$

As in the previous case, 


$$
\begin{aligned}
\mathcal{F}(\phi, \psi) & =\sum_{l=1}^{k} \sum_{j \neq j_{t}: 1 \leq t \leq k}\left(\left|\phi_{c_{j}} \phi_{c_{j_{l}}}\right|-\left|\phi_{c_{0}} \phi_{c_{j_{l}}}\right|\right) \\
& +\sum_{i \neq j, i \neq j_{t}, j \neq j_{t}: 1 \leq t \leq k}\left(\left|\phi_{c_{i}} \phi_{c_{j}}\right|-\left|\phi_{c_{0}} \phi_{c_{i j}}\right|\right) \\
& +\sum_{l \neq t}\left(\left|\phi_{c_{j_{t}}} \phi_{c_{j_{l}}}\right|-\left|\phi_{c_{0}} \phi_{c_{j_{t} j_{l}}}\right|\right)-(N-2) \sum_{i}\left|\phi_{c_{i}}\right|^{2} \\
& =X+Y
\end{aligned}
$$

where $X$ is the summand with $i=j_{l}$ and $j \neq j_{t}$ or $j=j_{l}$ and $i \neq j_{t}(1 \leq t \leq k$ and $l=1,2, \ldots, k)$; $Y$ is the summand with $i \neq j_{l}$ and $j \neq j_{l}$ or $i=j_{l}, j=j_{t}$. Then,

$$
X=\sum_{l=1}^{k} \sum_{j \neq j_{t}: 1 \leq t \leq k}\left(\left|\phi_{c_{j}} \phi_{c_{j_{l}}}\right|-\left|\phi_{c_{0}} \phi_{c_{j j_{l}}}\right|\right)<2 \sqrt{(N-1)} \cdot C_{\gamma}(\phi)
$$

and

$$
\begin{aligned}
Y & =\sum_{i \neq j_{t}, j \neq j_{t}: 1 \leq t \leq k}\left(\left|\phi_{c_{i}} \phi_{c_{j}}\right|-\left|\phi_{c_{0}} \phi_{c_{i j}}\right|\right)+\sum_{l \neq t}\left(\left|\phi_{c_{j_{t}}} \phi_{c_{j_{l}}}\right|-\left|\phi_{c_{0}} \phi_{c_{j_{t} j_{l}}}\right|\right)-(N-2) \sum_{i}\left|\phi_{c_{i}}\right|^{2} \\
& \leq \sum_{i \neq j_{t}, j \neq j_{t}: 1 \leq t \leq k} \frac{\left|\phi_{c_{i}}\right|^{2}+\left|\phi_{c_{j}}\right|^{2}}{2}+\sum_{l \neq t} \frac{\left|\phi_{c_{j_{j}}}\right|^{2}+\left|\phi_{c_{j_{t}}}\right|^{2}}{2}-(N-2) \sum_{i}\left|\phi_{c_{i}}\right|^{2} \\
& \leq(N-k-1) \sum_{i \neq j_{t}: 1 \leq t \leq k}\left(\left|\phi_{c_{i}}\right|^{2}\right)+(k-1) \sum_{l}\left(\left|\phi_{c_{j_{l}}}\right|^{2}\right)-(N-2) \sum_{i}\left|\phi_{c_{i}}\right|^{2} \\
& \leq(N-2) \sum_{i}\left|\phi_{c_{i}}\right|^{2}-(N-2) \sum_{i}\left|\phi_{c_{i}}\right|^{2} \\
& =0 .
\end{aligned}
$$

Combining together the two cases above, we conclude that

$$
\mathcal{F}(\phi, \psi) \leq \min _{\gamma} 2 \sqrt{(N-1)} \cdot C_{\gamma}(\phi)=2 \sqrt{(N-1)} \cdot C_{G M E}(\phi) .
$$

This ends the proof of the result stated in Eq. (5). The bound for mixed stated is given directly by the convexity of the GME concurrence (property M3 in Section 1) as follows. The bounds in Eqs. (6) and Eq. (77) can be easily obtained in analogous way, as it will be detailed in the next subsections.

Let

$$
\begin{aligned}
c_{0} & :=x_{1} \cdots x_{i-1} x_{i} x_{i+1} \cdots x_{N} \\
c_{i} & :=x_{1} \cdots x_{i-1} x_{i}^{\prime} x_{i+1} \cdots x_{N} \\
c_{j} & :=x_{1} \cdots x_{j-1} x_{j}^{\prime} x_{j+1} \cdots x_{N} \\
c_{i j} & :=x_{1} \cdots x_{i-1} x_{i}^{\prime} x_{i+1} \cdots x_{j-1} x_{j}^{\prime} x_{j+1} \cdots x_{N}
\end{aligned}
$$

when $i<j$, we use $c_{i j}$; otherwise, we use $c_{j i}$.

Let $\rho=\sum_{i} t_{k} \rho^{(k)}$ be the optimal decomposition of $\rho$ for the GME-concurrence, i.e, $C_{G M E}(\rho)=\sum_{k} t_{k} \cdot C_{G M E}\left(\rho^{(k)}\right)$, then

$$
\begin{aligned}
\mathcal{F}(\rho, \psi) & =\sum_{1 \leq i \neq j \leq N}\left|\rho_{c_{i}, c_{j}}\right|-\sum_{1 \leq i \neq j \leq N} \sqrt{\rho_{c_{0}, c_{0}} \rho_{c_{i j}, c_{i j}}}-(N-2) \sum_{1 \leq i \leq N} \rho_{c_{i}, c_{i}} \\
& =\sum_{1 \leq i \neq j \leq N}\left|\sum_{k} t_{k} \rho_{c_{i}, c_{j}}^{(k)}\right|-\sum_{1 \leq i \neq j \leq N} \sqrt{\sum_{k} t_{k} \rho_{c_{0}, c_{0}}^{(k)} \sum_{k} t_{k} \rho_{c_{i j}, c_{i j}}^{(k)}}-(N-2) \sum_{1 \leq i \leq N} \sum_{k} t_{k} \rho_{c_{i}, c_{i}}^{(k)}
\end{aligned}
$$


For the first term,

$$
\sum_{1 \leq i \neq j \leq N}\left|\sum_{k} t_{k} \rho_{c_{i}, c_{j}}^{(k)}\right| \leq \sum_{k} t_{k} \sum_{1 \leq i \neq j \leq N}\left|\rho_{c_{i}, c_{j}}^{(k)}\right|
$$

For the second term,

$$
\sqrt{\sum_{k} t_{k} \rho_{c_{0}, c_{0}}^{(k)} \sum_{k} t_{k} \rho_{c_{i j}, c_{i j}}^{(k)}}=\sqrt{\sum_{k_{1}, k_{2}} t_{k_{1}} t_{k_{2}} \rho_{c_{0}, c_{0}}^{\left(k_{1}\right)} \rho_{c_{i j}, c_{i j}}^{\left(k_{2}\right)}} \geq \sum_{k} t_{k} \sqrt{\rho_{c_{0}, c_{0}}^{(k)}} \sqrt{\rho_{c_{i j}, c_{i j}}^{(k)}}
$$

Therefore

$$
\sum_{1 \leq i \neq j \leq N} \sqrt{\sum_{k} t_{k} \rho_{c_{0}, c_{0}}^{(k)} \sum_{k} t_{k} \rho_{c_{i j}, c_{i j}}^{(k)}} \geq \sum_{1 \leq i \neq j \leq N} \sum_{k} t_{k} \sqrt{\rho_{c_{0}, c_{0}}^{(k)}} \sqrt{\rho_{c_{i j}, c_{i j}}^{(k)}}=\sum_{k} t_{k} \sum_{1 \leq i \neq j \leq N} \sqrt{\rho_{c_{0}}^{(k)}, c_{0}} \sqrt{\rho_{c_{i j}, c_{i j}}^{(k)}}
$$

Finally, for the third term,

$$
\sum_{1 \leq i \leq N} \sum_{k} t_{k} \rho_{c_{i}, c_{i}}^{(k)}=\sum_{k} t_{k} \sum_{1 \leq i \leq N} \rho_{c_{i}, c_{i}}^{(k)}
$$

Putting everything together, we obtain the bound in the statement:

$$
\begin{aligned}
\mathcal{F}(\rho, \psi) & \leq \sum_{k} t_{k} \sum_{1 \leq i \neq j \leq N}\left|\rho_{c_{i}, c_{j}}^{(k)}\right|-\sum_{k} t_{k} \sum_{1 \leq i \neq j \leq N} \sqrt{\rho_{c_{0}, c_{0}}^{(k)}} \sqrt{\rho_{c_{i j}, c_{i j}}^{(k)}}-(N-2) \sum_{k} t_{k} \sum_{1 \leq i \leq N} \rho_{c_{i}, c_{i}}^{(k)} \\
& =\sum_{k} t_{k}\left(\sum_{1 \leq i \neq j \leq N}\left|\rho_{c_{i}, c_{j}}^{(k)}\right|-\sum_{1 \leq i \neq j \leq N} \sqrt{\rho_{c_{0}, c_{0}}^{(k)}} \sqrt{\rho_{c_{i j}, c_{i j}}^{(k)}}-(N-2) \sum_{1 \leq i \leq N} \rho_{c_{i}, c_{i}}^{(k)}\right) \\
& \leq 2 \sqrt{(N-1)} \sum_{k} t_{k} \cdot C_{G M E}\left(\rho^{(k)}\right)=2 \sqrt{(N-1)} \cdot C_{G M E}(\rho)
\end{aligned}
$$

\section{Bound 2}

As we have already done above, we start with a warm-up case. It will be a four-qubit state. For a four-qubit state, $|\psi\rangle=|0000\rangle$, we obtain $\left|\psi_{1}\right\rangle=|1000\rangle,\left|\psi_{2}\right\rangle=|0100\rangle,\left|\psi_{3}\right\rangle=|0010\rangle$, and $\left|\psi_{4}\right\rangle=|0001\rangle$. We shall prove that

$$
\mathcal{L}\left(\rho, \psi_{1}\right)+\mathcal{L}\left(\rho, \psi_{2}\right)+\mathcal{L}\left(\rho, \psi_{3}\right)+\mathcal{L}\left(\rho, \psi_{4}\right) \leq 2 \sqrt{2} \cdot C_{G M E}(\rho)
$$

where

$$
\begin{aligned}
\mathcal{L}\left(\rho, \psi_{1}\right) & =2\left(\left|\rho_{10,11}\right|+\left|\rho_{10,13}\right|+\left|\rho_{11,13}\right|-\sqrt{\rho_{9,9} \rho_{12,12}}-\sqrt{\rho_{9,9} \rho_{14,14}}-\sqrt{\rho_{9,9} \rho_{15,15}}\right)-\left(\left|\rho_{10,10}\right|+\left|\rho_{11,11}\right|+\left|\rho_{13,13}\right|\right) \\
& =L_{11}+L_{12}-L_{13} ; \\
\mathcal{L}\left(\rho, \psi_{2}\right) & =2\left(\left|\rho_{6,7}\right|+\left|\rho_{6,13}\right|+\left|\rho_{7,13}\right|-\sqrt{\rho_{5,5} \rho_{8,8}}-\sqrt{\rho_{5,5} \rho_{14,14}}-\sqrt{\rho_{5,5} \rho_{15,15}}\right)-\left(\left|\rho_{6,6}\right|+\left|\rho_{7,7}\right|+\left|\rho_{13,13}\right|\right) \\
& =L_{21}+L_{22}-L_{23} ; \\
\mathcal{L}\left(\rho, \psi_{3}\right) & =2\left(\left|\rho_{4,7}\right|+\left|\rho_{4,11}\right|+\left|\rho_{7,11}\right|-\sqrt{\rho_{3,3} \rho_{8,8}}-\sqrt{\rho_{3,3} \rho_{12,12}}-\sqrt{\rho_{3,3} \rho_{15,15}}\right)-\left(\left|\rho_{4,4}\right|+\left|\rho_{7,7}\right|+\left|\rho_{11,11}\right|\right) \\
& =L_{31}+L_{32}-L_{33} ; \\
\mathcal{L}\left(\rho, \psi_{4}\right) & =2\left(\left|\rho_{4,6}\right|+\left|\rho_{4,10}\right|+\left|\rho_{6,10}\right|-\sqrt{\rho_{2,2} \rho_{8,8}}-\sqrt{\rho_{2,2} \rho_{12,12}}-\sqrt{\rho_{2,2} \rho_{14,14}}\right)-\left(\left|\rho_{4,4}\right|+\left|\rho_{6,6}\right|+\left|\rho_{10,10}\right|\right) \\
& =L_{41}+L_{42}-L_{43} .
\end{aligned}
$$

where $L_{i j}(1 \leq i \leq 4,1 \leq j \leq 3)$ depend on the partition. Each $L_{i j}$ is given in Appendix A. When $\rho$ is a pure state, that is $\rho=|\phi\rangle\langle\phi|$, for $C_{1}(\phi)$, we have the following: 


$$
\begin{aligned}
& L_{21}=2\left(\left|\phi_{0101} \phi_{1100}\right|+\left|\phi_{0110} \phi_{1100}\right|-\left|\phi_{0100} \phi_{1101}\right|-\left|\phi_{0100} \phi_{1110}\right|\right) ; \\
& L_{22}=\left(2\left|\phi_{0101} \phi_{0110}\right|-2\left|\phi_{0100} \phi_{0111}\right|-\left|\phi_{0101}\right|^{2}-\left|\phi_{0110}\right|^{2}\right) ; \\
& L_{23}=\left|\phi_{1100}\right|^{2} ; \\
& L_{31}=2\left(\left|\phi_{0011} \phi_{1010}\right|+\left|\phi_{0110} \phi_{1010}\right|-\left|\phi_{0010} \phi_{1011}\right|-\left|\phi_{0010} \phi_{1110}\right|\right) ; \\
& L_{32}=\left(2\left|\phi_{0011} \phi_{0110}\right|-2\left|\phi_{0010} \phi_{0111}\right|-\left|\phi_{0011}\right|^{2}-\left|\phi_{0110}\right|^{2}\right) ; \\
& L_{33}=\left|\phi_{1010}\right|^{2} ; \\
& L_{41}=2\left(\left|\phi_{0011} \phi_{1001}\right|+\left|\phi_{0101} \phi_{1001}\right|-\left|\phi_{0001} \phi_{1011}\right|-\left|\phi_{0001} \phi_{1101}\right|\right) ; \\
& L_{42}=\left(2\left|\phi_{0011} \phi_{0101}\right|-2\left|\phi_{0001} \phi_{0111}\right|-\left|\phi_{0011}\right|^{2}-\left|\phi_{0101}\right|^{2}\right) ; \\
& L_{43}=\left|\phi_{1001}\right|^{2} .
\end{aligned}
$$

It is obvious that $L_{22}, L_{32}, L_{42} \leq 0, L_{21}+L_{31}+L_{41} \leq 2 \sqrt{2} C_{1}(\rho)$, and $\mathcal{L}\left(\phi, \psi_{1}\right)-\left|\phi_{1001}\right|^{2}-\left|\phi_{1010}\right|^{2}-\left|\phi_{1100}\right|^{2} \leq 0$. Hence,

$$
\mathcal{L}\left(\phi, \psi_{1}\right)+\mathcal{L}\left(\phi, \psi_{2}\right)+\mathcal{L}\left(\phi, \psi_{3}\right)+\mathcal{L}\left(\phi, \psi_{4}\right) \leq 2 \sqrt{2} C_{1}(\phi) .
$$

The same holds for $C_{2}(\phi), C_{3}(\phi), C_{4}(\phi), C_{12}(\phi), C_{13}(\phi)$, and $C_{14}(\phi)$. As a consequence, for a pure state,

$$
\begin{aligned}
& \mathcal{L}\left(\phi, \psi_{1}\right)+\mathcal{L}\left(\phi, \psi_{2}\right)+\mathcal{L}\left(\phi, \psi_{3}\right)+\mathcal{L}\left(\phi, \psi_{4}\right) \\
& \leq 2 \sqrt{2} \min \left\{C_{1}(\phi), C_{2}(\phi), C_{3}(\phi), C_{4}(\phi), C_{12}(\phi), C_{13}(\phi), C_{14}(\phi)\right\} \\
& =2 \sqrt{2} C_{G M E}(\phi) .
\end{aligned}
$$

If $\rho$ is a mixed state, the convex roof construction is bounded as

$$
2 \sqrt{2} C_{G M E}(\rho) \geq \inf _{\left\{p_{i},\left|\phi_{i}\right\rangle\right\}} \sum_{i} p_{i}\left(\mathcal{L}\left(\phi_{i}, \psi_{1}\right)+\mathcal{L}\left(\phi_{i}, \psi_{2}\right)+\mathcal{L}\left(\phi_{i}, \psi_{3}\right)+\mathcal{L}\left(\phi_{i}, \psi_{4}\right)\right),
$$

where $\left\{p_{i}, \phi^{(i)}\right\}$ is any pure state decomposition of $\rho$. Since $C_{G M E}(\rho)$ is invariant under local unitaries, for any choice of a product state $\left|\psi_{1}\right\rangle, \mathcal{L}\left(\rho, \psi_{1}\right)$ is a lower bound to $C_{G M E}(\rho)$ leading to $2 \sqrt{2} C_{G M E}(\rho) \geq\left(\mathcal{L}\left(\rho, \psi_{1}\right)+\mathcal{L}\left(\rho, \psi_{2}\right)+\right.$ $\left.\mathcal{L}\left(\rho, \psi_{3}\right)+\mathcal{L}\left(\rho, \psi_{4}\right)\right)$. This concludes the proof for the four-qubit case.

We are now ready to prove the inequality for a general $N$-qubit state. If

$$
c_{i j k}:=x_{1} \cdots x_{i-1} x_{i}^{\prime} x_{i+1} \cdots x_{j-1} x_{j}^{\prime} x_{j+1} \cdots x_{k-1} x_{k}^{\prime} x_{k+1} \cdots x_{N}
$$

then

$$
\mathcal{L}\left(\phi, \psi_{c_{i}}\right)=\sum_{j \neq i, k \neq i, j \neq k} 2\left(\left|\phi_{c_{i j}} \phi_{c_{i k}}\right|-\left|\phi_{c_{0}} \phi_{c_{i j k}}\right|\right)-(N-3) \sum_{1 \leq j \leq N, j \neq i}\left|\phi_{c_{i j}}\right|^{2} .
$$

There are again two cases in close analogy with the previus part.

Case 1. For any given $\gamma \subset\{1,2, \ldots N\}$ with $|\{\gamma\}|=1$,

$$
\begin{aligned}
2 \sqrt{N-2} C_{\gamma}(\phi) & =2 \sqrt{N-2} \sqrt{\sum_{j \neq \gamma, i \neq \gamma, j \neq i}\left|\phi_{c_{i \gamma}} \phi_{c_{i j}}-\phi_{c_{i}} \phi_{c_{i j \gamma}}\right|^{2}} \\
& \geq \sum_{j \neq \gamma, i \neq \gamma, j \neq i}\left|\phi_{c_{i \gamma}} \phi_{c_{i j}}-\phi_{c_{i}} \phi_{c_{i j \gamma}}\right| \geq \sum_{j \neq \gamma, i \neq \gamma, j \neq i}\left|\phi_{c_{i \gamma}} \phi_{c_{i j}}\right|-\left|\phi_{c_{i}} \phi_{c_{i j \gamma}}\right|,
\end{aligned}
$$

Now,

$$
\begin{aligned}
\mathcal{L}\left(\phi, \psi_{i}\right) & =\sum_{j \neq \gamma, i \neq \gamma, j \neq i} 2\left(\left|\phi_{c_{i j}} \phi_{c_{i \gamma}}\right|-\left|\phi_{c_{i}} \phi_{c_{i j \gamma}}\right|\right)+\sum_{i \neq \gamma, j \neq \gamma, k \neq \gamma, i \neq j, i \neq k} 2\left(\left|\phi_{c_{i j}} \phi_{c_{i k}}\right|-\left|\phi_{c_{i}} \phi_{c_{i j k}}\right|\right) \\
& -(N-3) \sum_{1 \leq j \leq N, j \neq i, j \neq \gamma}\left|\phi_{c_{i j}}\right|^{2}-(N-3)\left|\phi_{c_{i \gamma}}\right|^{2} \\
& =L_{i 1}+L_{i 2}-L_{i 3},
\end{aligned}
$$


where

$$
\begin{aligned}
L_{i 1} & =\sum_{j \neq \gamma, i \neq \gamma, j \neq i} 2\left(\left|\phi_{c_{i j}} \phi_{c_{i \gamma}}\right|-\left|\phi_{c_{i}} \phi_{c_{i j \gamma}}\right|\right) ; \\
L_{i 2} & =\sum_{i \neq \gamma, j \neq \gamma, k \neq \gamma, i \neq j} 2\left(\left|\phi_{c_{i j}} \phi_{c_{i k}}\right|-\left|\phi_{c_{i}} \phi_{c_{i j k}}\right|\right)-(N-3) \sum_{1 \leq j \leq N, j \neq i}\left|\phi_{c_{i j}}\right|^{2} \\
& \leq \sum_{i \neq \gamma, j \neq \gamma, k \neq \gamma, i \neq j}\left(\left|\phi_{c_{i j}}\right|^{2}+\left|\phi_{c_{i k}}\right|^{2}\right)-(N-3) \sum_{1 \leq j \leq N, j \neq i}\left|\phi_{c_{i j}}\right|^{2} \\
& =(N-3) \sum_{1 \leq j \leq N, j \neq i}\left|\phi_{c_{i j}}\right|^{2}-(N-3) \sum_{1 \leq j \leq N, j \neq i, j \neq \gamma}\left|\phi_{c_{i j}}\right|^{2} \leq 0 ; \\
L_{i 3} & =(N-3)\left|\phi_{c_{i \gamma}}\right|^{2} .
\end{aligned}
$$

Thus,

$$
\begin{aligned}
\sum_{1 \leq i \leq N, i \neq \gamma} L_{i 1} & \leq 2 \sqrt{2} C_{G M E}(\rho), \\
L_{i 2} & \leq 0, \text { for } 1 \leq i \leq N,
\end{aligned}
$$

and

$$
\mathcal{L}\left(\phi, \psi_{\gamma}\right)-\sum_{1 \leq i \leq N, i \neq \gamma} L_{i 3} \leq 0
$$

So, for pure states,

$$
\begin{aligned}
\mathcal{L}\left(\phi, \psi_{1}\right)+\mathcal{L}\left(\phi, \psi_{2}\right)+\mathcal{L}\left(\phi, \psi_{3}\right)+\mathcal{L}\left(\phi, \psi_{4}\right) & \leq 2 \sqrt{2} \min \left\{C_{1}(\phi), C_{2}(\phi), C_{3}(\phi), C_{4}(\phi), C_{12}(\phi), C_{13}(\phi), C_{14}(\phi)\right\} \\
& =2 \sqrt{2} C_{G M E}(\phi) .
\end{aligned}
$$

Case 2. For any given $\gamma=\left\{j_{1}, j_{2}, \ldots, j_{k}\right\} \subset\{1,2, \ldots N\}$, with $k \geq 2$,

$$
\begin{aligned}
2 \sqrt{N-2} \cdot C_{\gamma}(\phi) & \geq 2 \sqrt{N-2)} \cdot C_{\gamma}(\phi) \\
& =2 \sqrt{N-2} \sqrt{\sum_{l=1}^{k} \sum_{j \neq j_{t}: 1 \leq t \leq k}\left|\phi_{c_{i j_{l}}} \phi_{c_{i j}}-\phi_{c_{i}} \phi_{c_{i j_{l} j}}\right|^{2}} \geq \sum_{l=1}^{k} \sum_{j \neq j_{t}: 1 \leq t \leq k}\left|\phi_{c_{i j_{l}}} \phi_{c_{i j}}\right|-\left|\phi_{c_{i}} \phi_{c_{i j_{l} j}}\right|
\end{aligned}
$$

As in the previous case,

$$
\begin{aligned}
\mathcal{L}\left(\phi, \psi_{i}\right) & =\sum_{l=1}^{k} \sum_{j \neq j_{t}: 1 \leq t \leq k}\left(\left|\phi_{c_{i j}} \phi_{c_{i j_{l}}}\right|-\left|\phi_{c_{i}} \phi_{c_{i j j_{l}}}\right|\right)+\sum_{i \neq j, i \neq j_{t}, s \neq j_{t}, j \neq j_{t}: 1 \leq t \leq k}\left(\left|\phi_{c_{i s}} \phi_{c_{i j}}\right|-\left|\phi_{c_{i}} \phi_{c_{i j s}}\right|\right) \\
& +\sum_{l \neq t}\left(\left|\phi_{c_{i j_{t}}} \phi_{c_{i j_{l}}}\right|-\left|\phi_{c_{i}} \phi_{c_{i j_{t} j_{l}}}\right|\right)-(N-3) \sum_{1 \leq j \leq N, j \neq i}\left|\phi_{c_{i j}}\right|^{2} \\
& =L_{i 1}+L_{i 2},
\end{aligned}
$$

where $X$ is the summand with $i=j_{l}$ and $j \neq j_{t}$ or $j=j_{l}$ and $i \neq j_{t}(1 \leq t \leq k) ; Y$ is the summand with $i \neq j_{l}$ and $j \neq j_{l}(l=1,2, \ldots, k)$ or $i=j_{l}, j=j_{t}$. Then,

$$
L_{i 1}=\sum_{l=1}^{k} \sum_{j \neq j_{t}: 1 \leq t \leq k}\left(\left|\phi_{c_{i j}} \phi_{c_{i j_{l}}}\right|-\left|\phi_{c_{i}} \phi_{c_{i j j_{l}}}\right|\right)
$$


and

$$
\begin{aligned}
L_{i 2} & =\sum_{i \neq j, i \neq j_{t}, s \neq j_{t}, j \neq j_{t}: 1 \leq t \leq k}\left(\left|\phi_{c_{i s}} \phi_{c_{i j}}\right|-\left|\phi_{c_{i}} \phi_{c_{i j s}}\right|\right)+\sum_{l \neq t}\left(\left|\phi_{c_{i j_{t}}} \phi_{c_{i j_{l}}}\right|-\left|\phi_{c_{i}} \phi_{c_{i j_{t} j_{l}}}\right|\right) \\
& -(N-3) \sum_{1 \leq j \leq N, j \neq i}\left|\phi_{c_{i j}}\right|^{2} \\
& \leq \sum_{i \neq j, i \neq j_{t}, s \neq j_{t}, j \neq j_{t}: 1 \leq t \leq k} \frac{\left|\phi_{c_{i s}}\right|^{2}+\left|\phi_{c_{i j}}\right|^{2}}{2}+\sum_{l \neq t} \frac{\left|\phi_{c_{i j_{l}}}\right|^{2}+\left|\phi_{c_{i j_{t}}}\right|^{2}}{2}-(N-3) \sum_{1 \leq j \leq N, j \neq i}\left|\phi_{c_{i j}}\right|^{2} \\
& \leq(N-k-1) \sum_{i \neq s: 1 \leq t \leq k}\left(\left|\phi_{c_{i s}}\right|^{2}\right)+(k-2) \sum_{l}\left(\left|\phi_{c_{i j_{l}}}\right|^{2}\right) \\
& \leq(N-3) \sum_{i}\left|\phi_{c_{i}}\right|^{2}-(N-3) \sum_{i}\left|\phi_{c_{i}}\right|^{2} \\
& =0 .
\end{aligned}
$$

For an $n$-qubit state,

$$
\sum_{1 \leq i \leq N} \mathcal{L}\left(\phi, \psi_{i}\right) \leq 2 \sqrt{N-2} \min _{\gamma}\left\{C_{\gamma}(\phi)\right\}=2 \sqrt{N-2} C_{G M E}(\phi)
$$

\section{Bound 3}

Let $V=\left\{\left|\chi_{1}\right\rangle, \ldots,\left|\chi_{4}\right\rangle\right\}$ be a set of product states in $\mathcal{H}$, where $\left|\chi_{1}\right\rangle=|0011\rangle,\left|\chi_{2}\right\rangle=|0101\rangle,\left|\chi_{3}\right\rangle=|0110\rangle$, and $\left|\chi_{4}\right\rangle=|1010\rangle$. Let $K_{1}=\left\{\left|\chi_{2}\right\rangle,\left|\chi_{3}\right\rangle,\left|\chi_{4}\right\rangle\right\}, K_{2}=\left\{\left|\chi_{1}\right\rangle,\left|\chi_{3}\right\rangle\right\}, K_{3}=\left\{\left|\chi_{1}\right\rangle,\left|\chi_{2}\right\rangle,\left|\chi_{4}\right\rangle\right\}$, and $K_{4}=\left\{\left|\chi_{1}\right\rangle,\left|\chi_{3}\right\rangle\right\}$. Here, $s=3$. Details of the next steps are in Appendix B. If $\rho$ is a pure state, $\rho=|\phi\rangle\langle\phi|$, we can prove that Bound 3 is $\mathcal{T}(\rho, \chi) \leq 3 \sqrt{2} \cdot C_{G M E}(\rho)$. For any given $\gamma \subset\{1,2, \ldots N\}$ such that $|\{\gamma\}|=1$, take, e.g., $\gamma=\{1\}$, i.e., the case $C_{1}(\rho)$. Let $\mathcal{T}(\rho, \chi)=X+Y$. The sum giving $X$ involves terms $\left|\chi_{\alpha}\right\rangle$ and $\left|\chi_{\beta}\right\rangle$ that are different in qubit 1 ; on the other hand, the terms of $Y$ are the states $\left|\chi_{\alpha}\right\rangle$ and $\left|\chi_{\beta}\right\rangle$ that are different in the other qubits, except qubit 1 . This is $X \leq 3 \sqrt{2} C_{1}(\rho)$ and $Y \leq 0$ (see Appendix B.1). So $\mathcal{T}(\rho, \chi)=X+Y \leq 3 \sqrt{2} C_{1}(\rho)$. We can use the same process to get $\mathcal{T}(\rho, \chi)=X+Y \leq 3 \sqrt{2} C_{2}(\rho), \mathcal{T}(\rho, \chi)=X+Y \leq 3 \sqrt{2} C_{3}(\rho)$, and $\mathcal{T}(\rho, \chi)=X+Y \leq 3 \sqrt{2} C_{4}(\rho)$.

Now if $|\{\gamma\}|=2$, take $\gamma=\{1,2\}$ as the example, i.e., $C_{12}(\rho)$. Again, let $\mathcal{T}(\rho, \chi)=X+Y$.The sum giving $X$ involves terms $\left|\chi_{\alpha}\right\rangle$ and $\left|\chi_{\beta}\right\rangle$ that are different in only one of the $\gamma$ qubits; the terms of $Y$ are defined analogously to the previous case. Hence, $X \leq 3 \sqrt{2} C_{12}(\rho)$ and $Y \leq 0$ (see Appendix B.2). We obtain $\mathcal{T}(\rho, \chi)=X+Y \leq 3 \sqrt{2} C_{12}(\rho)$, and, by the same process, $\mathcal{T}(\rho, \chi)=X+Y \leq 3 \sqrt{2} C_{13}(\rho), \mathcal{T}(\rho, \chi)=X+Y \leq 3 \sqrt{2} C_{14}(\rho)$, and $\mathcal{T}(\rho, \chi) \leq 3 \sqrt{2} C_{G M E}(\rho)$.

For a mixed state $\rho$, the bound is given by using the convex roof construction. For the general case, the sum giving $\mathcal{T}(\rho, \chi)$ simplifies because only two qubits of $\left|\chi_{\alpha}\right\rangle$ and $\left|\chi_{\beta}\right\rangle$ are different. For any given $\gamma \subset\{1,2, \ldots N\}, \mathcal{T}(\rho, \chi)$ can be subdivided into two addends, which we will denote by $X$ and $Y$. If $|\{\gamma\}|=1$, the sum giving $X$ involves terms $\left|\chi_{\alpha}\right\rangle$ and $\left|\chi_{\beta}\right\rangle$ that are different in the $\gamma$ qubit; on the other hand, the terms of $Y$ are the states $\left|\chi_{\alpha}\right\rangle$ and $\left.\chi_{\beta}\right\rangle$ that are different in the other qubits except for the $\gamma$ qubit; the case $|\{\gamma\}|>1$ is an easy generalization. From this,

$$
X=\sum_{\mid \chi_{\alpha\rangle \in V}} \sum_{A}\left(\left|\left\langle\chi_{\alpha}|\rho| \chi_{\beta}\right\rangle\right|-\sqrt{\left\langle\chi_{\alpha}\left|\otimes\left\langle\chi_{\beta}\left|\Pi_{\alpha \beta} \rho^{\otimes 2} \Pi_{\alpha \beta}\right| \chi_{\alpha}\right\rangle \otimes\right| \chi_{\beta}\right\rangle}\right)
$$

and

$$
Y=\sum_{\mid \chi_{\alpha\rangle \in V}} \sum_{B}\left(\left|\left\langle\chi_{\alpha}|\rho| \chi_{\beta}\right\rangle\right|-\sqrt{\left\langle\chi_{\alpha}\left|\otimes\left\langle\chi_{\beta}\left|\Pi_{\alpha \beta} \rho^{\otimes 2} \Pi_{\alpha \beta}\right| \chi_{\alpha}\right\rangle \otimes\right| \chi_{\beta}\right\rangle}\right)-\left(s-s_{0}\right) \sum_{a}\left\langle\chi_{\alpha}|\rho| \chi_{\alpha}\right\rangle .
$$

When $\rho$ is a pure state, $\rho=|\phi\rangle\langle\phi|$, if $\left|\chi_{\alpha}\right\rangle$ and $\left|\chi_{\beta}\right\rangle$ are different in the $\gamma$ qubit only, the value $\mid\left\langle\chi_{\alpha}|\rho| \chi_{\beta}\right\rangle-$ $\sqrt{\left\langle\chi_{\alpha}\left|\otimes\left\langle\chi_{\beta}\left|\Pi_{\alpha \beta} \rho^{\otimes 2} \Pi_{\alpha \beta}\right| \chi_{\alpha}\right\rangle \otimes\right| \chi_{\beta}\right\rangle \mid}$ is one of the terms of $C_{\gamma}(\rho)$ and the maximum number of terms in $X$ is less than $2 s^{2}$; when all states in $K_{\alpha}$ belong to $A$ for any $\chi_{\alpha} \in V$, and for any $\alpha,\left|K_{\alpha}\right|=s$, the maximum number of terms in $X$ is $2(s+s-1+\cdots+1)=s(s+1) \leq 2 s^{2}$. So, $X \leq \sqrt{2} s C_{\gamma}(\rho)$. Concerning $Y$, for every $\left|\chi_{\alpha}\right\rangle$ in $V$, the maximum number of terms in the sum is $s-s_{0}$. For any $\alpha$, the maximum number of the terms $\left|\left\langle\chi_{\alpha}|\rho| \chi_{\beta}\right\rangle\right|$ is $s$, but 
the minimum number of terms $\left|\left\langle\chi_{\alpha}|\rho| \chi_{\beta}\right\rangle\right|$ in $X$ is $s_{0}$, and so the maximum number of terms in $Y$ is $s-s_{0}$. Hence, $Y \leq 0$ and $X+Y \leq \sqrt{2} s C_{\gamma}(\rho)$. For a mixed state $\rho$, the convex roof construction is bounded as

$$
\sqrt{2} s C_{G M E}(\rho) \geq \inf _{\left\{p_{i},\left|\phi_{i}\right\rangle\right\}} \sum_{i} p_{i} \mathcal{T}\left(\phi_{i}, \psi\right),
$$

where $\left\{p_{i}, \phi^{(i)}\right\}$ is any pure state decomposition of $\rho$. Consequently we can get the desired result that $\mathcal{T}(\rho, \chi) \leq$ $\sqrt{2} s C_{G M E}(\rho)$.

\section{EXAMPLES}

In this section, we illustrate our main result with some explicit examples. The first one is useful to clarify the bounds:

Example V.1 Given $|\phi\rangle=|0000\rangle$, we obtain $\left|\phi_{1}\right\rangle=|1000\rangle,\left|\phi_{2}\right\rangle=|0100\rangle,\left|\phi_{3}\right\rangle=|0010\rangle$, and $\left|\phi_{4}\right\rangle=|0001\rangle$, by applying the bit flip operation. The bound in Eq. (6) is $\mathcal{L}\left(\rho, \phi_{1}\right)+\mathcal{L}\left(\rho, \phi_{2}\right)+\mathcal{L}\left(\rho, \phi_{3}\right)+\mathcal{L}\left(\rho, \phi_{4}\right) \leq 2 \sqrt{2} \cdot C_{G M E}(\rho)$, where

$$
\begin{aligned}
& \mathcal{L}\left(\rho, \phi_{1}\right)=2\left(\left|\rho_{10,11}\right|+\left|\rho_{10,13}\right|+\left|\rho_{11,13}\right|-\sqrt{\rho_{9,9} \rho_{12,12}}-\sqrt{\rho_{9,9} \rho_{14,14}}-\sqrt{\rho_{9,9} \rho_{15,15}}\right)-\left(\rho_{10,10}+\rho_{11,11}+\rho_{13,13}\right) ; \\
& \mathcal{L}\left(\rho, \phi_{2}\right)=2\left(\left|\rho_{6,7}\right|+\left|\rho_{6,13}\right|+\left|\rho_{7,13}\right|-\sqrt{\rho_{5,5} \rho_{8,8}}-\sqrt{\rho_{5,5} \rho_{14,14}}-\sqrt{\rho_{5,5} \rho_{15,15}}\right)-\left(\rho_{6,6}+\rho_{7,7}+\rho_{13,13}\right) ; \\
& \mathcal{L}\left(\rho, \phi_{3}\right)=2\left(\left|\rho_{4,7}\right|+\left|\rho_{4,11}\right|+\left|\rho_{7,11}\right|-\sqrt{\rho_{3,3} \rho_{8,8}}-\sqrt{\rho_{3,3} \rho_{12,12}}-\sqrt{\rho_{3,3} \rho_{15,15}}\right)-\left(\rho_{4,4}+\rho_{7,7}+\rho_{11,11}\right) ; \\
& \mathcal{L}\left(\rho, \phi_{4}\right)=2\left(\left|\rho_{4,6}\right|+\left|\rho_{4,10}\right|+\left|\rho_{6,10}\right|-\sqrt{\rho_{2,2} \rho_{8,8}}-\sqrt{\rho_{2,2} \rho_{12,12}}-\sqrt{\rho_{2,2} \rho_{14,14}}\right)-\left(\rho_{4,4}+\rho_{6,6}+\rho_{10,10}\right) .
\end{aligned}
$$

For the bound in Eq. (7), let us fix $\left|v_{1}\right\rangle=|0011\rangle,\left|v_{2}\right\rangle=|0101\rangle,\left|v_{3}\right\rangle=|0110\rangle$, and $\left|v_{4}\right\rangle=|1010\rangle$. Let $K_{1}=$ $\left\{\left|v_{2}\right\rangle,\left|v_{3}\right\rangle,\left|v_{4}\right\rangle\right\}, K_{2}=\left\{\left|v_{1}\right\rangle,\left|v_{3}\right\rangle\right\}, K_{3}=\left\{\left|v_{1}\right\rangle,\left|v_{2}\right\rangle,\left|v_{4}\right\rangle\right\}$, and $K_{4}=\left\{\left|v_{1}\right\rangle,\left|v_{3}\right\rangle\right\}(s=3)$. Then $2\left(\left|\rho_{4,6}\right|+\left|\rho_{4,7}\right|+\left|\rho_{4,11}\right|+\right.$ $\left.\left|\rho_{6,7}\right|+\left|\rho_{7,11}\right|-\sqrt{\rho_{2,2} \rho_{8,8}}-\sqrt{\rho_{8,8} \rho_{5,5}}-\sqrt{\rho_{3,3} \rho_{5,5}}-\sqrt{\rho_{3,3} \rho_{12,12}}-\sqrt{\rho_{3,3} \rho_{15,15}}\right)-2\left(\rho_{4,4}+\rho_{6,6}+\rho_{7,7}+\rho_{11,11}\right) \leq \sqrt{6} \cdot C_{G M E}(\rho)$. Example V.2 Let us consider a two-parameter four-qubit state given by a mixture of the identity matrix, the $W$ state, and the anti- $W$ state:

$$
\rho=\frac{1-a-b}{32} I_{32}+a|\widetilde{W}\rangle\langle\widetilde{W}|+b| W\rangle\langle W|
$$

with $|W\rangle=\frac{1}{\sqrt{5}}(|00001\rangle+|00010\rangle+|00100\rangle+|01000\rangle+|10000\rangle)$ and $|\widetilde{W}\rangle=\frac{1}{\sqrt{5}}(|11110\rangle+|11101\rangle+|11011\rangle+|10111\rangle+$ $|01111\rangle$ ). Fig. (11) illustrates the GME area detected by the Bound 1 (Section [V A) and Eq. (III) in [13], respectively. The area detected by the former is visibly larger.

Our Bound 1 is as follows:

$$
\begin{aligned}
4 \sqrt{2} C_{G M E}(\rho) \geq & 2\left(\left|\rho_{2,3}\right|+\left|\rho_{2,5}\right|+\left|\rho_{2,9}\right|+\left|\rho_{2,17}\right|+\left|\rho_{3,5}\right|+\left|\rho_{3,9}\right|+\left|\rho_{3,17}\right|+\left|\rho_{5,9}\right|+\left|\rho_{5,17}\right|+\left|\rho_{9,17}\right|\right. \\
& -\sqrt{\rho_{1,1} \rho_{4,4}}-\sqrt{\rho_{1,1} \rho_{6,6}}-\sqrt{\rho_{1,1} \rho_{10,10}}-\sqrt{\rho_{1,1} \rho_{18,18}}-\sqrt{\rho_{1,1} \rho_{7,7}} \\
& \left.-\sqrt{\rho_{1,1} \rho_{11,11}}-\sqrt{\rho_{1,1} \rho_{19,19}}-\sqrt{\rho_{1,1} \rho_{13,13}}-\sqrt{\rho_{1,1} \rho_{21,21}}-\sqrt{\rho_{1,1} \rho_{25,25}}\right) \\
& -3\left(\rho_{2,2}+\rho_{3,3}+\rho_{5,5}+\rho_{9,9}+\rho_{17,17}\right) ;
\end{aligned}
$$

$$
\begin{aligned}
4 \sqrt{2} C_{G M E}(\rho) \geq 2 & \left(\left|\rho_{16,24}\right|+\left|\rho_{16,28}\right|+\left|\rho_{16,30}\right|+\left|\rho_{16,31}\right|+\left|\rho_{24,28}\right|+\left|\rho_{24,30}\right|+\left|\rho_{24,31}\right|+\left|\rho_{28,30}\right|+\left|\rho_{28,31}\right|+\left|\rho_{30,31}\right|\right. \\
& -\sqrt{\rho_{32,32} \rho_{8,8}}-\sqrt{\rho_{32,32} \rho_{12,12}}-\sqrt{\rho_{32,32} \rho_{14,14}}-\sqrt{\rho_{32,32} \rho_{15,15}}-\sqrt{\rho_{32,32} \rho_{20,20}} \\
& \left.-\sqrt{\rho_{32,32} \rho_{22,22}}-\sqrt{\rho_{32,32} \rho_{23,23}}-\sqrt{\rho_{32,32} \rho_{26,26}}-\sqrt{\rho_{32,32} \rho_{27,27}}-\sqrt{\rho_{32,32} \rho_{29,29}}\right) \\
& -3\left(\rho_{16,16}+\rho_{24,24}+\rho_{28,28}+\rho_{30,30}+\rho_{31,31}\right) .
\end{aligned}
$$

The above two equations give bounds to $C_{G M E}(\rho)$. From these, we get $\frac{67 b+35 a-35}{64} \leq 4 \sqrt{2} C_{G M E}(\rho)$ and $\frac{67 a+35 b-35}{64} \leq 4 \sqrt{2} C_{G M E}(\rho)$, respectively. The entanglement area above the lowest line is obtained by taking $\frac{67 b+35 a-35}{64}>0$ or $\frac{67 a+35 b-35}{64}>0$. The entanglement area is the union set of $\left\{(a, b) \mid \frac{67 b+35 a-35}{64}>0\right\}$ and $\left\{(a, b) \mid \frac{67 a+35 b-35}{64}>0\right\}$. The bound of Eq. (III) in [13] gives instead

$$
\begin{aligned}
4 \sqrt{2} C_{G M E}(\rho) \geq 2 & \left(\left|\rho_{2,3}\right|+\left|\rho_{2,5}\right|+\left|\rho_{2,9}\right|+\left|\rho_{2,17}\right|+\left|\rho_{3,5}\right|+\left|\rho_{3,9}\right|+\left|\rho_{3,17}\right|+\left|\rho_{5,9}\right|+\left|\rho_{5,17}\right|+\left|\rho_{9,17}\right|\right) \\
& -3\left(2 \sqrt{\rho_{1,1} \rho_{4,4}}+2 \sqrt{\rho_{1,1} \rho_{6,6}}+2 \sqrt{\rho_{1,1} \rho_{10,10}}+2 \sqrt{\rho_{1,1} \rho_{18,18}}\right. \\
& +2 \sqrt{\rho_{1,1} \rho_{7,7}}+2 \sqrt{\rho_{1,1} \rho_{11,11}}+2 \sqrt{\rho_{1,1} \rho_{19,19}}+2 \sqrt{\rho_{1,1} \rho_{13,13}} \\
& \left.+2 \sqrt{\rho_{1,1} \rho_{21,21}}+2 \sqrt{\rho_{1,1} \rho_{25,25}}+\rho_{2,2}+\rho_{3,3}+\rho_{5,5}+\rho_{9,9}+\rho_{17,17}\right) ;
\end{aligned}
$$




$$
\begin{aligned}
4 \sqrt{2} C_{G M E}(\rho) \geq 2 & \left(\left|\rho_{16,24}\right|+\left|\rho_{16,28}\right|+\left|\rho_{16,30}\right|+\left|\rho_{16,31}\right|+\left|\rho_{24,28}\right|+\left|\rho_{24,30}\right|+\left|\rho_{24,31}\right|+\left|\rho_{28,30}\right|+\left|\rho_{28,31}\right|+\left|\rho_{30,31}\right|\right) \\
& -3\left(2 \sqrt{\rho_{32,32} \rho_{8,8}}+2 \sqrt{\rho_{32,32} \rho_{12,12}}+2 \sqrt{\rho_{32,32} \rho_{14,14}}+2 \sqrt{\rho_{32,32} \rho_{15,15}}\right. \\
& +2 \sqrt{\rho_{32,32} \rho_{20,20}}+2 \sqrt{\rho_{32,32} \rho_{22,22}}+2 \sqrt{\rho_{32,32} \rho_{23,23}}+2 \sqrt{\rho_{32,32} \rho_{26,26}} \\
& \left.+2 \sqrt{\rho_{32,32 \rho_{27,27}}}+2 \sqrt{\rho_{32,32 \rho_{29,29}}}+\rho_{16,16}+\rho_{24,24}+\rho_{28,28}+\rho_{30,30}+\rho_{31,31}\right) .
\end{aligned}
$$

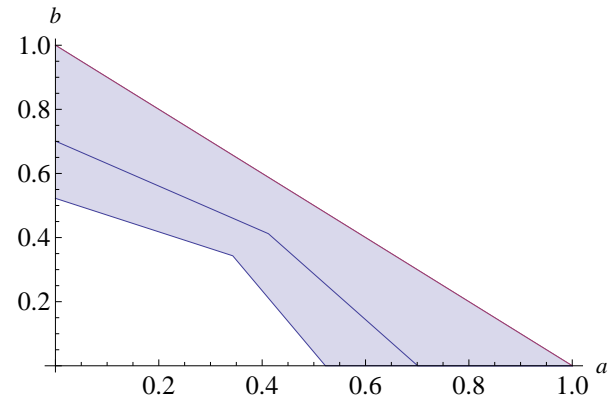

FIG. 1: The entanglement area of the density matrix $\rho$ in Eq. 9 detected by Bound 1. The area is above the lowest line. The area detected by Eq. (III) in [13] is above the middle line.

From these equations, we have $\frac{75 a+107 b-75}{32}$ and $\frac{75 b+107 a-75}{32}$, respectively. The entanglement area above the middle line is obtained by $\frac{75 a+107 b-75}{32}>0$ or $\frac{75 b+107 b-75}{32}>0$.

Example V.3 Let us consider the one-parameter four-qubit state $\rho=\frac{1-a}{16} I_{16}+a|\phi\rangle\langle\phi|$, with $|\phi\rangle=\frac{1}{2}(|0011\rangle+|0101\rangle+$ $|0110\rangle+|1010\rangle)$. Bound 3, Eq. (77) $)$ gives the bound $2\left(\left|\rho_{4,6}\right|+\left|\rho_{4,11}\right|-\sqrt{\rho_{2,2} \rho_{8,8}}-\sqrt{\rho_{3,3} \rho_{12,12}}\right)-\left(\rho_{4,4}+\rho_{6,6}+\rho_{11,11}\right)$. This shows that GME area is $a>\frac{7}{11}$. On the other hand, by making use of a criterion in [15] or our Eq. (6), we can find that there is GME for $a>\frac{9}{11}$.

In the following examples, the method in [16] and Eq. (III) in [13] can not detect entanglement at all.

Example V.4 Let us consider the one-parameter three-qutrit state $\rho=\frac{1-a}{27} I_{27}+a|\phi\rangle\langle\phi|$, with $|\phi\rangle=\frac{1}{\sqrt{3}}(|012\rangle+$ $|021\rangle+|111\rangle)$. Bound 1, Eq. (5) indicates that there is GME for any $a>\frac{1}{4}$. Also, to give a four-qubit example, let $\rho=\frac{1-a}{16} I_{16}+a|\phi\rangle\langle\phi|$, with $|\phi\rangle=\frac{1}{2}(|0000\rangle+|1100\rangle+|1001\rangle+|1010\rangle)$. Our result detects GME for $a>\frac{5}{9}$.

Example V.5 Let us consider the one-parameter four-qubit state $\rho=\frac{1-a}{16} I_{16}+a|\phi\rangle\langle\phi|$, with $|\phi\rangle=\frac{1}{\sqrt{5}}(|0000\rangle+$ $|1100\rangle+|1001\rangle+|1010\rangle+|0110\rangle)$. By applying Eq. (5), we can see that the GME area is $a>\frac{25}{41}=0.60976$. However, Eq. (17) (Bound 3) detects GME for $a>\frac{45}{61}=0.7377$.

Example V.6 Let us consider the one-parameter four-qubits state $\rho=\frac{1-a}{16} I_{16}+a\left|\phi_{0112}^{4}\right\rangle\left\langle\phi_{0112}^{4}|,| \phi_{0112}^{4}\right\rangle=\frac{1}{\sqrt{6}}(|1100\rangle+$ $|0110\rangle+|1001\rangle+|0101\rangle+|1010\rangle+|0011\rangle)$. The density matrix $\rho$ is a mixture of white noise and the Dicke state [17]. By applying Bound 2 given in Eq. (6),$a>\frac{9}{17}$.

\section{CONCLUSIONS}

We have studied genuine multipartite entanglement of quantum states. We have given an alternative definition of pure state GME-concurrence based on the expectation value of an observable with respect to a two-fold copy of the state under consideration. This definition has the advantage of being physical accessible (e.g., with the use of twin photons [14, 18] ). We have then proposed several analytical lower bounds for GME-concurrence. Such bounds are also given as expectation values of some observable. On the basis of the bounds, we have obtained entanglement criteria that can be used to detect GME for states of generic dimension. We have reported examples in which the criteria perform better than the previously known methods.

Note added. Recently, we became aware of Ref.[19], where the authors also derive similar results.

Acknowledgment. ZHM is supported by NSF of China (10901103) and by the Foundation of China Scholarship Council (2010831012). JLC is supported by NSF of China (10975075) and the Fundamental Research Funds for the 
Central Universities. SS is supported by the Royal Society. Part of this work has been carried out while SS was visiting the Institute of Natural Sciences at Shanghai Jiao-Tong University. The financial support of this institution is gratefully acknowledged. While finishing our manuscript we became aware of [19], where the authors also derive similar results. We are indebted to Marcus Huber for carefully reading earlier drafts and for many important comments that helped improving the paper.

[1] R. Raussendorf and H.-J. Briegel, Phys. Rev. Lett. 86, 5188 (2001).

[2] S. Schauer, M. Huber, and B. C. Hiesmayr, Phys. Rev. A 82, 062311 (2010).

[3] D. Markham and B. C. Sanders, Phys. Rev. A 78, 042309 (2008).

[4] W. Dür, G. Vidal, and J. I. Cirac, Phys. Rev. A 62, 062314 (2000).

[5] J. T. Barreiro, P. Schindler, O. Gühne, T. Monz, M. Chwalla, C. F. Roos, M. Hennrich, R. Blatt, Nat. Phys. 6, 943 (2010).

[6] B. Jungnitsch, T. Moroder, and O. Gühne, Phys. Rev. Lett. 106, 190502 (2011).

[7] J.-D. Bancal, N. Gisin, Y.-C. Liang, and S. Pironio, Phys. Rev. Lett. 106, 250404 (2011).

[8] R. Horodecki, P. Horodecki, M. Horodecki, and K. Horodecki, Rev. Mod. Phys. 81, 865 (2009).

[9] O. Gühne, G. Toth, Phys. Rep. 474, 1(2009).

[10] V. Coffman, J. Kundu and W. K. Wootters, Phys. Rev. A 61, 052306 (2000).

[11] Z.-H. Ma, Z.-H. Chen, J.-L. Chen, C. Spengler, A. Gabriel, and M. Huber, Phys. Rev. A 83, 062325 (2011).

[12] L. Aolita, F. Mintert, Phys. Rev. Lett. 97, 050501(2006).

[13] M. Huber, F. Mintert, A. Gabriel, and B. C. Hiesmayr, Phys. Rev. Lett. 104, 210501 (2010).

[14] O. Gühne and M. P. Seevinck, New J. Phys. 12, 053002 (2010).

[15] M. Huber, P. Erker, H. Schimpf, A. Gabriel, and B. C. Hiesmayr, Phys. Rev. A 83, 040301(R) (2011).

[16] T. Gao, Y. Hong, Phys. Rev. A 82, 062113 (2010).

[17] R. H. Dicke, Phys. Rev. 93, 99 (1954).

[18] S. P. Walborn, P. H. Souto Ribeiro, L. Davidovich, F. Mintert, and A. Buchleitner, Nature(London) 440, 1022 (2006).

[19] J. Wu, H. Kampermann, D. Bruß, C. Klöckl, M. Huber, Experimentally feasible lower bounds on measures of multipartite entanglement, arXiv:1205.3119.

\section{Appendix A: Proof of Bound 2: details}

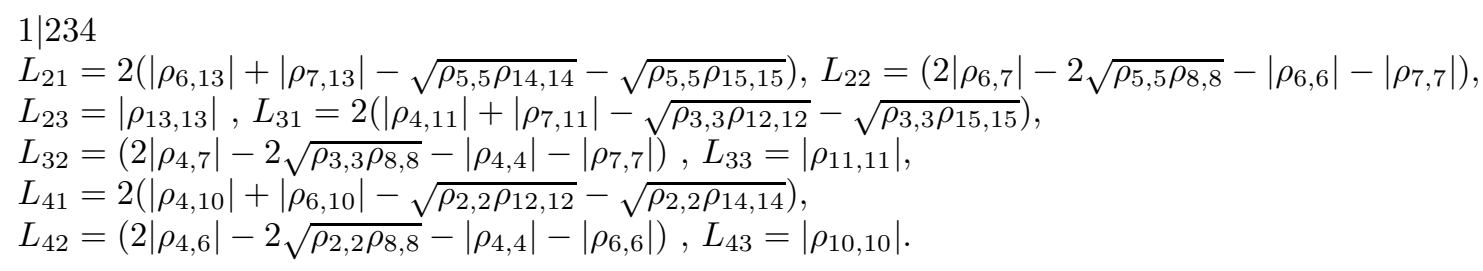

$$
\begin{aligned}
& 2 \mid 134 \\
& L_{11}=2\left(\left|\rho_{10,11}\right|+\left|\rho_{11,13}\right|-\sqrt{\rho_{9,9} \rho_{12,12}}-\sqrt{\rho_{9,9} \rho_{15,15}}\right), \\
& L_{12}=\left(2\left|\rho_{10,13}\right|-2 \sqrt{\rho_{9,9} \rho_{14,14}}-\left|\rho_{10,10}\right|-\left|\rho_{13,13}\right|\right), L_{13}=\left|\rho_{11,11}\right|, \\
& L_{31}=2\left(\left|\rho_{4,7}\right|+\left|\rho_{7,11}\right|-\sqrt{\rho_{3,3} \rho_{8,8}}-\sqrt{\rho_{3,3} \rho_{15,15}}\right), \\
& L_{32}=\left(2\left|\rho_{4,11}\right|-2 \sqrt{\rho_{3,3} \rho_{12,12}}-\left|\rho_{4,4}\right|-\left|\rho_{11,11}\right|\right), L_{33}=\left|\rho_{7,7}\right|, \\
& L_{41}=2\left(\left|\rho_{4,6}\right|+\left|\rho_{6,10}\right|-\sqrt{\rho_{2,2} \rho_{8,8}}-\sqrt{\rho_{2,2} \rho_{14,14}}\right), \\
& L_{42}=\left(2\left|\rho_{4,10}\right|-2 \sqrt{\rho_{2,2} \rho_{12,12}}-\left|\rho_{4,4}\right|-\left|\rho_{10,10}\right|\right), L_{43}=\left|\rho_{6,6}\right| .
\end{aligned}
$$

$3 \mid 124$

$$
\begin{aligned}
& L_{11}=2\left(\left|\rho_{10,13}\right|+\left|\rho_{11,13}\right|-\sqrt{\rho_{9,9} \rho_{14,14}}-\sqrt{\rho_{9,9} \rho_{15,15}}\right), \\
& L_{12}=\left(2\left|\rho_{10,11}\right|-2 \sqrt{\rho_{9,9} \rho_{12,12}}-\left|\rho_{10,10}\right|-\left|\rho_{11,11}\right|\right), L_{13}=\left|\rho_{13,13}\right|, \\
& L_{21}=2\left(\left|\rho_{6,7}\right|+\left|\rho_{7,13}\right|-\sqrt{\rho_{5,5} \rho_{8,8}}-\sqrt{\rho_{5,5} \rho_{15,15}}\right), \\
& L_{22}=\left(2\left|\rho_{6,13}\right|-2 \sqrt{\rho_{5,5} \rho_{14,14}}-\left|\rho_{6,6}\right|-\left|\rho_{13,13}\right|\right), L_{23}=\left|\rho_{7,7}\right|, \\
& L_{41}=2\left(\left|\rho_{4,6}\right|+\left|\rho_{4,10}\right|-\sqrt{\rho_{2,2} \rho_{8,8}}-\sqrt{\rho_{2,2} \rho_{12,12}}\right), \\
& L_{42}=\left(2\left|\rho_{6,10}\right|-2 \sqrt{\rho_{2,2} \rho_{14,14}}-\left|\rho_{6,6}\right|-\left|\rho_{10,10}\right|\right), L_{43}=\left|\rho_{4,4}\right| .
\end{aligned}
$$


$4 \mid 123$

$L_{11}=2\left(\left|\rho_{10,13}\right|+\left|\rho_{11,13}\right|-\sqrt{\rho_{9,9} \rho_{14,14}}-\sqrt{\rho_{9,9} \rho_{15,15}}\right)$,

$L_{12}=\left(2\left|\rho_{10,11}\right|-2 \sqrt{\rho_{9,9} \rho_{12,12}}-\left|\rho_{10,10}\right|-\left|\rho_{11,11}\right|\right), L_{13}=\left|\rho_{13,13}\right|$,

$L_{21}=2\left(\left|\rho_{6,7}\right|+\left|\rho_{7,13}\right|-\sqrt{\rho_{5,5} \rho_{8,8}}-\sqrt{\rho_{5,5} \rho_{15,15}}\right)$,

$L_{22}=\left(2\left|\rho_{6,13}\right|-2 \sqrt{\rho_{5,5} \rho_{14,14}}-\left|\rho_{6,6}\right|-\left|\rho_{13,13}\right|\right), L_{23}=\left|\rho_{7,7}\right|$,

$L_{31}=2\left(\left|\rho_{4,7}\right|+\left|\rho_{4,11}\right|-\sqrt{\rho_{3,3} \rho_{8,8}}-\sqrt{\rho_{3,3} \rho_{12,12}}\right)$,

$L_{32}=\left(2\left|\rho_{7,11}\right|-2 \sqrt{\rho_{3,3} \rho_{15,15}}-\left|\rho_{7,7}\right|-\left|\rho_{11,11}\right|\right), L_{33}=\left|\rho_{4,4}\right|$.

$12 \mid 34$

$L_{11}=2\left(\left|\rho_{10,13}\right|+\left|\rho_{11,13}\right|-\sqrt{\rho_{9,9} \rho_{14,14}}-\sqrt{\rho_{9,9} \rho_{15,15}}\right)$,

$L_{12}=\left(2\left|\rho_{10,11}\right|-2 \sqrt{\rho_{9,9} \rho_{12,12}}-\left|\rho_{10,10}\right|-\left|\rho_{11,11}\right|\right), L_{13}=\left|\rho_{13,13}\right|$,

$L_{21}=2\left(\left|\rho_{6,7}\right|+\left|\rho_{7,13}\right|-\sqrt{\rho_{5,5} \rho_{8,8}}-\sqrt{\rho_{5,5} \rho_{15,15}}\right)$,

$L_{22}=\left(2\left|\rho_{6,13}\right|-2 \sqrt{\rho_{5,5} \rho_{14,14}}-\left|\rho_{6,6}\right|-\left|\rho_{13,13}\right|\right), L_{23}=\left|\rho_{7,7}\right|$,

$L_{31}=2\left(\left|\rho_{4,7}\right|+\left|\rho_{7,11}\right|-\sqrt{\rho_{3,3} \rho_{8,8}}-\sqrt{\rho_{3,3} \rho_{15,15}}\right)$,

$L_{32}=\left(2\left|\rho_{4,11}\right|-2 \sqrt{\rho_{3,3} \rho_{12,12}}-\left|\rho_{4,4}\right|-\left|\rho_{11,11}\right|\right), L_{33}=\left|\rho_{7,7}\right|$,

$L_{41}=2\left(\left|\rho_{4,6}\right|+\left|\rho_{6,10}\right|-\sqrt{\rho_{2,2} \rho_{8,8}}-\sqrt{\rho_{2,2} \rho_{12,12}}\right)$,

$L_{42}=\left(2\left|\rho_{4,10}\right|-2 \sqrt{\rho_{2,2} \rho_{14,14}}-\left|\rho_{4,4}\right|-\left|\rho_{10,10}\right|\right), L_{43}=\left|\rho_{6,6}\right|$.

$13 \mid 24$

$L_{11}=2\left(\left|\rho_{10,11}\right|+\left|\rho_{11,13}\right|-\sqrt{\rho_{9,9} \rho_{12,12}}-\sqrt{\rho_{9,9} \rho_{15,15}}\right)$,

$L_{12}=\left(2\left|\rho_{10,13}\right|-2 \sqrt{\rho_{9,9} \rho_{14,14}}-\left|\rho_{10,10}\right|-\left|\rho_{13,13}\right|\right), L_{13}=\left|\rho_{11,11}\right|$,

$L_{21}=2\left(\left|\rho_{6,7}\right|+\left|\rho_{6,13}\right|-\sqrt{\rho_{5,5} \rho_{8,8}}-\sqrt{\rho_{5,5} \rho_{14,14}}\right)$,

$L_{22}=\left(2\left|\rho_{7,13}\right|-2 \sqrt{\rho_{5,5} \rho_{15,15}}-\left|\rho_{7,7}\right|-\left|\rho_{13,13}\right|\right), L_{23}=\left|\rho_{6,6}\right|$,

$L_{31}=2\left(\left|\rho_{4,11}\right|+\left|\rho_{7,11}\right|-\sqrt{\rho_{3,3} \rho_{12,12}}-\sqrt{\rho_{3,3} \rho_{15,15}}\right)$,

$L_{32}=\left(2\left|\rho_{4,7}\right|-2 \sqrt{\rho_{3,3} \rho_{8,8}}-\left|\rho_{4,4}\right|-\left|\rho_{7,7}\right|\right), L_{33}=\left|\rho_{11,11}\right|$,

$L_{41}=2\left(\left|\rho_{4,6}\right|+\left|\rho_{6,10}\right|-\sqrt{\rho_{2,2} \rho_{8,8}}-\sqrt{\rho_{2,2} \rho_{12,12}}\right)$,

$L_{42}=\left(2\left|\rho_{4,10}\right|-2 \sqrt{\rho_{2,2} \rho_{14,14}}-\left|\rho_{4,4}\right|-\left|\rho_{10,10}\right|\right), L_{43}=\left|\rho_{6,6}\right|$.

$14 \mid 23$

$L_{11}=2\left(\left|\rho_{10,11}\right|+\left|\rho_{10,13}\right|-\sqrt{\rho_{9,9} \rho_{12,12}}-\sqrt{\rho_{9,9} \rho_{14,14}}\right)$,

$L_{12}=\left(2\left|\rho_{11,13}\right|-2 \sqrt{\rho_{9,9} \rho_{15,15}}-\left|\rho_{11,11}\right|-\left|\rho_{13,13}\right|\right), L_{13}=\left|\rho_{10,10}\right|$,

$L_{21}=2\left(\left|\rho_{6,7}\right|+\left|\rho_{7,13}\right|-\sqrt{\rho_{5,5} \rho_{8,8}}-\sqrt{\rho_{5,5} \rho_{15,15}}\right)$,

$L_{22}=\left(2\left|\rho_{6,13}\right|-2 \sqrt{\rho_{5,5} \rho_{14,14}}-\left|\rho_{6,6}\right|-\left|\rho_{13,13}\right|\right), L_{23}=\left|\rho_{7,7}\right|$,

$L_{31}=2\left(\left|\rho_{4,7}\right|+\left|\rho_{7,11}\right|-\sqrt{\rho_{3,3} \rho_{8,8}}-\sqrt{\rho_{3,3} \rho_{15,15}}\right)$,

$L_{32}=\left(2\left|\rho_{4,11}\right|-2 \sqrt{\rho_{3,3} \rho_{12,12}}-\left|\rho_{4,4}\right|-\left|\rho_{11,11}\right|\right), L_{33}=\left|\rho_{7,7}\right|$,

$L_{41}=2\left(\left|\rho_{4,10}\right|+\left|\rho_{6,10}\right|-\sqrt{\rho_{2,2} \rho_{14,14}}-\sqrt{\rho_{2,2} \rho_{12,12}}\right)$,

$L_{42}=\left(2\left|\rho_{4,6}\right|-2 \sqrt{\rho_{2,2} \rho_{8,8}}-\left|\rho_{4,4}\right|-\left|\rho_{6,6}\right|\right), L_{43}=\left|\rho_{10,10}\right|$. 


\section{Appendix B: Proof of Bound 3: details}

1. $C_{1}(\rho)$

$$
\begin{aligned}
\mathcal{T}(\rho, \chi) & =\left|\left\langle\chi_{1}|\rho| \chi_{2}\right\rangle\right|-\sqrt{\left\langle\chi_{1}\left|\otimes\left\langle\chi_{2}\left|\Pi_{12} \rho^{\otimes 2} \Pi_{12}\right| \chi_{1}\right\rangle \otimes\right| \chi_{2}\right\rangle}+\left|\left\langle\chi_{1}|\rho| \chi_{3}\right\rangle\right|-\sqrt{\left\langle\chi_{1}\left|\otimes\left\langle\chi_{3}\left|\Pi_{13} \rho^{\otimes 2} \Pi_{13}\right| \chi_{1}\right\rangle \otimes\right| \chi_{3}\right\rangle} \\
& +\left|\left\langle\chi_{1}|\rho| \chi_{4}\right\rangle\right|-\sqrt{\left\langle\chi_{1}\left|\otimes\left\langle\chi_{4}\left|\Pi_{14} \rho^{\otimes 2} \Pi_{14}\right| \chi_{1}\right\rangle \otimes\right| \chi_{4}\right\rangle}+\left|\left\langle\chi_{2}|\rho| \chi_{1}\right\rangle\right|-\sqrt{\left\langle\chi_{2}\left|\otimes\left\langle\chi_{1}\left|\Pi_{12} \rho^{\otimes 2} \Pi_{12}\right| \chi_{2}\right\rangle \otimes\right| \chi_{1}\right\rangle} \\
& +\left|\left\langle\chi_{2}|\rho| \chi_{3}\right\rangle\right|-\sqrt{\left\langle\chi_{2}\left|\otimes\left\langle\chi_{3}\left|\Pi_{23} \rho^{\otimes 2} \Pi_{23}\right| \chi_{2}\right\rangle \otimes\right| \chi_{3}\right\rangle}+\left|\left\langle\chi_{3}|\rho| \chi_{1}\right\rangle\right|-\sqrt{\left\langle\chi_{3}\left|\otimes\left\langle\chi_{1}\left|\Pi_{13} \rho^{\otimes 2} \Pi_{13}\right| \chi_{3}\right\rangle \otimes\right| \chi_{1}\right\rangle} \\
& +\left|\left\langle\chi_{3}|\rho| \chi_{2}\right\rangle\right|-\sqrt{\left\langle\chi_{3}\left|\otimes\left\langle\chi_{2}\left|\Pi_{23} \rho^{\otimes 2} \Pi_{23}\right| \chi_{3}\right\rangle \otimes\right| \chi_{2}\right\rangle}+\left|\left\langle\chi_{3}|\rho| \chi_{4}\right\rangle\right|-\sqrt{\left\langle\chi_{3}\left|\otimes\left\langle\chi_{4}\left|\Pi_{34} \rho^{\otimes 2} \Pi_{34}\right| \chi_{3}\right\rangle \otimes\right| \chi_{4}\right\rangle} \\
& \left.+\left|\left\langle\chi_{4}|\rho| \chi_{1}\right\rangle\right|-\sqrt{\left\langle\chi_{4}\left|\otimes\left\langle\chi_{1}\left|\Pi_{14} \rho^{\otimes 2} \Pi_{14}\right| \chi_{4}\right\rangle \otimes\right| \chi_{1}\right\rangle}+\left|\left\langle\chi_{4}|\rho| \chi_{3}\right\rangle\right|-\sqrt{\left\langle\chi_{4}\left|\otimes\left\langle\chi_{3}\left|\Pi_{34} \rho^{\otimes 2} \Pi_{34}\right| \chi_{4}\right\rangle \otimes\right| \chi_{3}\right\rangle}\right) \\
& =2\left(\left|\left\langle\chi_{1}|\rho| \chi_{2}\right\rangle\right|-\sqrt{\left\langle\chi_{1}\left|\otimes\left\langle\chi_{2}\left|\Pi_{12} \rho^{\otimes 2} \Pi_{12}\right| \chi_{1}\right\rangle \otimes\right| \chi_{2}\right\rangle}+\left|\left\langle\chi_{1}|\rho| \chi_{3}\right\rangle\right|-\sqrt{\left\langle\chi_{1}\left|\otimes\left\langle\chi_{3}\left|\Pi_{13} \rho^{\otimes 2} \Pi_{13}\right| \chi_{1}\right\rangle \otimes\right| \chi_{3}\right\rangle}\right. \\
& +\left|\left\langle\chi_{1}|\rho| \chi_{4}\right\rangle\right|-\sqrt{\left\langle\chi_{1}\left|\otimes\left\langle\chi_{4}\left|\Pi_{14} \rho^{\otimes 2} \Pi_{14}\right| \chi_{1}\right\rangle \otimes\right| \chi_{4}\right\rangle}+\left|\left\langle\chi_{2}|\rho| \chi_{3}\right\rangle\right|-\sqrt{\left\langle\chi_{2}\left|\otimes\left\langle\chi_{3}\left|\Pi_{23} \rho^{\otimes 2} \Pi_{23}\right| \chi_{2}\right\rangle \otimes\right| \chi_{3}\right\rangle} \\
& +\left|\left\langle\chi_{3}|\rho| \chi_{4}\right\rangle\right|-\sqrt{\left\langle\chi_{3}\left|\otimes\left\langle\chi_{4}\left|\Pi_{34} \rho^{\otimes 2} \Pi_{34}\right| \chi_{3}\right\rangle \otimes\right| \chi_{4}\right\rangle} \\
& =2\left(\left|\phi_{0011} \phi_{0101}\right|-\left|\phi_{0001} \phi_{0111}\right|+\left|\phi_{0011} \phi_{0110}\right|-\left|\phi_{0010} \phi_{1110}\right|+\left|\phi_{0011} \phi_{1010}\right|-\left|\phi_{0010} \phi_{1011}\right|+\left|\phi_{0110} \phi_{0101}\right|\right. \\
& \left.-\left|\phi_{0100} \phi_{0111}\right|+\left|\phi_{0110} \phi_{1010}\right|-\left|\phi_{0010} \phi_{1110}\right|\right)-2\left(\left|\phi_{0011}\right|^{2}+\left|\phi_{0101}\right|^{2}+\left|\phi_{0110}\right|^{2}+\left|\phi_{1010}\right|^{2}\right) \\
& \leq 3 \sqrt{2} \cdot C_{G M E}(\rho)
\end{aligned}
$$

$$
\begin{aligned}
X & =\left|\left\langle\chi_{1}|\rho| \chi_{4}\right\rangle\right|-\sqrt{\left\langle\chi_{1}\left|\otimes\left\langle\chi_{4}\left|\Pi_{14} \rho^{\otimes 2} \Pi_{14}\right| \chi_{1}\right\rangle \otimes\right| \chi_{4}\right\rangle}+\left|\left\langle\chi_{3}|\rho| \chi_{4}\right\rangle\right|-\sqrt{\left\langle\chi_{3}\left|\otimes\left\langle\chi_{4}\left|\Pi_{34} \rho^{\otimes 2} \Pi_{34}\right| \chi_{3}\right\rangle \otimes\right| \chi_{4}\right\rangle} \\
& +\left|\left\langle\chi_{4}|\rho| \chi_{1}\right\rangle\right|-\sqrt{\left\langle\chi_{4}\left|\otimes\left\langle\chi_{1}\left|\Pi_{14} \rho^{\otimes 2} \Pi_{14}\right| \chi_{4}\right\rangle \otimes\right| \chi_{1}\right\rangle}+\left|\left\langle\chi_{4}|\rho| \chi_{3}\right\rangle\right|-\sqrt{\left\langle\chi_{4}\left|\otimes\left\langle\chi_{3}\left|\Pi_{34} \rho^{\otimes 2} \Pi_{34}\right| \chi_{4}\right\rangle \otimes\right| \chi_{3}\right\rangle} \\
& =2\left(\left|\phi_{0011} \phi_{1010}\right|-\left|\phi_{1011} \phi_{0010}\right|+\left|\phi_{0110} \phi_{1010}\right|-\left|\phi_{1110} \phi_{0010}\right|\right) \\
& \leq 2\left(\left|\phi_{0011} \phi_{1010}-\phi_{1011} \phi_{0010}\right|+\left|\phi_{0110} \phi_{1010}-\phi_{1110} \phi_{0010}\right|\right) \\
& \leq 3 \sqrt{2} C_{1}(\rho) ;
\end{aligned}
$$

$$
\begin{aligned}
Y & =\left|\left\langle\chi_{1}|\rho| \chi_{2}\right\rangle\right|-\sqrt{\left\langle\chi_{1}\left|\otimes\left\langle\chi_{2}\left|\Pi_{12} \rho^{\otimes 2} \Pi_{12}\right| \chi_{1}\right\rangle \otimes\right| \chi_{2}\right\rangle}+\left|\left\langle\chi_{1}|\rho| \chi_{3}\right\rangle\right|-\sqrt{\left\langle\chi_{1}\left|\otimes\left\langle\chi_{3}\left|\Pi_{13} \rho^{\otimes 2} \Pi_{13}\right| \chi_{1}\right\rangle \otimes\right| \chi_{3}\right\rangle} \\
& +\left|\left\langle\chi_{2}|\rho| \chi_{1}\right\rangle\right|-\sqrt{\left\langle\chi_{2}\left|\otimes\left\langle\chi_{1}\left|\Pi_{12} \rho^{\otimes 2} \Pi_{12}\right| \chi_{2}\right\rangle \otimes\right| \chi_{1}\right\rangle}+\left|\left\langle\chi_{2}|\rho| \chi_{3}\right\rangle\right|-\sqrt{\left\langle\chi_{2}\left|\otimes\left\langle\chi_{3}\left|\Pi_{23} \rho^{\otimes 2} \Pi_{23}\right| \chi_{2}\right\rangle \otimes\right| \chi_{3}\right\rangle} \\
& +\left|\left\langle\chi_{3}|\rho| \chi_{1}\right\rangle\right|-\sqrt{\left\langle\chi_{3}\left|\otimes\left\langle\chi_{1}\left|\Pi_{13} \rho^{\otimes 2} \Pi_{13}\right| \chi_{3}\right\rangle \otimes\right| \chi_{1}\right\rangle}+\left|\left\langle\chi_{3}|\rho| \chi_{2}\right\rangle\right|-\sqrt{\left\langle\chi_{3}\left|\otimes\left\langle\chi_{2}\left|\Pi_{23} \rho^{\otimes 2} \Pi_{23}\right| \chi_{3}\right\rangle \otimes\right| \chi_{2}\right\rangle} \\
& =2\left(\left|\phi_{0011} \phi_{0101}\right|-\left|\phi_{0001} \phi_{0111}\right|+\left|\phi_{0011} \phi_{0110}\right|-\left|\phi_{0010} \phi_{0111}\right|+\left|\phi_{0110} \phi_{0101}\right|-\left|\phi_{0100} \phi_{0111}\right|\right) \\
& -2\left(\left|\phi_{0011}\right|^{2}+\left|\phi_{0101}\right|^{2}+\left|\phi_{0110}\right|^{2}+\left|\phi_{1010}\right|^{2}\right) \\
& \leq 2\left(\left|\phi_{0011} \phi_{0101}\right|+\left|\phi_{0011} \phi_{0110}\right|+\left|\phi_{0110} \phi_{0101}\right|\right)-2\left(\left|\phi_{0011}\right|^{2}+\left|\phi_{0101}\right|^{2}+\left|\phi_{0110}\right|^{2}+\left|\phi_{1010}\right|^{2}\right) \\
& \leq 0 .
\end{aligned}
$$

2. $C_{12}(\rho)$

$$
\begin{aligned}
X & =\left|\left\langle\chi_{1}|\rho| \chi_{2}\right\rangle\right|-\sqrt{\left\langle\chi_{1}\left|\otimes\left\langle\chi_{2}\left|\Pi_{12} \rho^{\otimes 2} \Pi_{12}\right| \chi_{1}\right\rangle \otimes\right| \chi_{2}\right\rangle}+\left|\left\langle\chi_{1}|\rho| \chi_{3}\right\rangle\right|-\sqrt{\left\langle\chi_{1}\left|\otimes\left\langle\chi_{3}\left|\Pi_{13} \rho^{\otimes 2} \Pi_{13}\right| \chi_{1}\right\rangle \otimes\right| \chi_{3}\right\rangle} \\
& +\left|\left\langle\chi_{1}|\rho| \chi_{4}\right\rangle\right|-\sqrt{\left\langle\chi_{1}\left|\otimes\left\langle\chi_{4}\left|\Pi_{14} \rho^{\otimes 2} \Pi_{14}\right| \chi_{1}\right\rangle \otimes\right| \chi_{4}\right\rangle}+\left|\left\langle\chi_{2}|\rho| \chi_{1}\right\rangle\right|-\sqrt{\left\langle\chi_{2}\left|\otimes\left\langle\chi_{1}\left|\Pi_{12} \rho^{\otimes 2} \Pi_{12}\right| \chi_{2}\right\rangle \otimes\right| \chi_{1}\right\rangle} \\
& +\left|\left\langle\chi_{3}|\rho| \chi_{1}\right\rangle\right|-\sqrt{\left\langle\chi_{3}\left|\otimes\left\langle\chi_{1}\left|\Pi_{13} \rho^{\otimes 2} \Pi_{13}\right| \chi_{3}\right\rangle \otimes\right| \chi_{1}\right\rangle}+\left|\left\langle\chi_{4}|\rho| \chi_{1}\right\rangle\right|-\sqrt{\left\langle\chi_{4}\left|\otimes\left\langle\chi_{1}\left|\Pi_{14} \rho^{\otimes 2} \Pi_{14}\right| \chi_{4}\right\rangle \otimes\right| \chi_{1}\right\rangle} \\
& =2\left(\left|\phi_{0011} \phi_{0101}\right|-\left|\phi_{0001} \phi_{0111}\right|+\left|\phi_{0011} \phi_{0110}\right|-\left|\phi_{0010} \phi_{0111}\right|+\left|\phi_{0011} \phi_{1010}\right|-\left|\phi_{1011} \phi_{0010}\right|\right) \\
& \leq 2\left(\left|\phi_{0011} \phi_{0101}-\phi_{0001} \phi_{0111}\right|+\left|\phi_{0011} \phi_{0110}-\phi_{0010} \phi_{0111}\right|+\left|\phi_{0011} \phi_{1010}-\phi_{1011} \phi_{0010}\right|\right) \\
& \leq 3 \sqrt{2} C_{12}(\rho)
\end{aligned}
$$




$$
\begin{aligned}
Y & =\left|\left\langle\chi_{2}|\rho| \chi_{3}\right\rangle\right|-\sqrt{\left\langle\chi_{2}\left|\otimes\left\langle\chi_{3}\left|\Pi_{23} \rho^{\otimes 2} \Pi_{23}\right| \chi_{2}\right\rangle \otimes\right| \chi_{3}\right\rangle}+\left|\left\langle\chi_{3}|\rho| \chi_{2}\right\rangle\right|-\sqrt{\left\langle\chi_{3}\left|\otimes\left\langle\chi_{2}\left|\Pi_{23} \rho^{\otimes 2} \Pi_{23}\right| \chi_{3}\right\rangle \otimes\right| \chi_{2}\right\rangle} \\
& +\left|\left\langle\chi_{3}|\rho| \chi_{4}\right\rangle\right|-\sqrt{\left\langle\chi_{3}\left|\otimes\left\langle\chi_{4}\left|\Pi_{34} \rho^{\otimes 2} \Pi_{34}\right| \chi_{3}\right\rangle \otimes\right| \chi_{4}\right\rangle}+\left|\left\langle\chi_{4}|\rho| \chi_{3}\right\rangle\right|-\sqrt{\left\langle\chi_{4}\left|\otimes\left\langle\chi_{3}\left|\Pi_{34} \rho^{\otimes 2} \Pi_{34}\right| \chi_{4}\right\rangle \otimes\right| \chi_{3}\right\rangle} \\
& =2\left(\left|\phi_{0110} \phi_{0101}\right|-\left|\phi_{0100} \phi_{0111}\right|+\left|\phi_{0110} \phi_{1010}\right|-\left|\phi_{1110} \phi_{0010}\right|\right) \\
& -2\left(\left|\phi_{0011}\right|^{2}+\left|\phi_{0101}\right|^{2}+\left|\phi_{0110}\right|^{2}+\left|\phi_{1010}\right|^{2}\right) \\
& \leq 2\left(\left|\phi_{0110} \phi_{0101}\right|+\left|\phi_{0110} \phi_{1010}\right|\right)-2\left(\left|\phi_{0011}\right|^{2}+\left|\phi_{0101}\right|^{2}+\left|\phi_{0110}\right|^{2}+\left|\phi_{1010}\right|^{2}\right) \\
& \leq 0 .
\end{aligned}
$$

